\title{
The Rate of Convergence to the Asymptotics for the Wave Equation in an Exterior Domain
}

\author{
By \\ Soichiro Katayama and Hideo Kubo \\ (Wakayama University and Tohoku University, Japan) \\ Dedicated to Professor Tatsuo Nishitani on the occasion of his 60th birthday
}

\begin{abstract}
In this paper we consider the mixed problem for the wave equation exterior to a non-trapping obstacle in odd space dimensions. We derive a rate of the convergence of the solution for the mixed problem to its asymptotic profile, which is written as a solution for the Cauchy problem. As a by-product, we are able to find out the radiation field of solutions to the mixed problem in terms of the scattering data.

Key Words and Phrases. Wave equation, Exterior problem, Asymptotic behavior, Convergence rate.

2000 Mathematics Subject Classification Numbers. 35L20; 35 B40.
\end{abstract}

\section{Introduction}

This paper is concerned with the global behavior of solutions to the mixed problem for the wave equation in an exterior domain:

$$
\begin{aligned}
& \left(\partial_{t}^{2}-\Delta\right) u(t, x)=0, \quad(t, x) \in(0, \infty) \times \Omega, \\
& u(t, x)=0, \quad(t, x) \in(0, \infty) \times \partial \Omega, \\
& u(0, x)=f_{0}(x),\left(\partial_{t} u\right)(0, x)=f_{1}(x), \quad x \in \Omega,
\end{aligned}
$$

where $\Omega=\boldsymbol{R}^{n} \backslash \overline{\mathcal{O}}$, and $\mathcal{O}$ is a bounded open set in $\boldsymbol{R}^{n}$ with smooth boundary. Throughout this paper, we suppose that $n$ is an odd integer with $n \geq 3$. We assume that $\Omega$ is connected and that the initial data $\vec{f}=\left(f_{0}, f_{1}\right)$ belongs to the associated energy space $\mathscr{H}_{D}(\Omega)$. Here and in the following, for an open set $Y \subset \boldsymbol{R}^{n}, \mathscr{H}_{D}(Y)$ stands for the completion of $\left(C_{0}^{\infty}(Y)\right)^{2}$ with respect to $\|\vec{f}\|_{\mathscr{H}_{D}(Y)}=\left\|\nabla f_{0}\right\|_{L^{2}(Y)}+\left\|f_{1}\right\|_{L^{2}(Y)} . \quad U(t)$ denotes the propagator of the mixed problem (1.1) with (1.2) and (1.3); in other words, for each $t \in \boldsymbol{R}$ we define a unitary operator $U(t)$ on $\mathscr{H}_{D}(\Omega)$ by

$$
U(t) \vec{f}=\left(u(t, \cdot), \partial_{t} u(t, \cdot)\right)
$$

for $\vec{f} \in \mathscr{H}_{D}(\Omega)$, where $u$ is the solution to $(1.1)-(1.3)$. 
It is well known that the solution to the above problem asymptotically approaches a solution to the Cauchy problem. More precisely, for a given initial data $\vec{f} \in \mathscr{H}_{D}(\Omega)$ there exists uniquely a scattering data $\vec{f}_{+} \in \mathscr{H}_{D}\left(\boldsymbol{R}^{n}\right)$ such that

$$
\left\|U(t) \vec{f}-\left.\left(U_{0}(t) \vec{f}_{+}\right)\right|_{\Omega}\right\|_{\mathscr{H}_{D}(\Omega)} \rightarrow 0 \quad(t \rightarrow \infty),
$$

where $U_{0}(t)$ is a unitary operator on $\mathscr{H}_{D}\left(\boldsymbol{R}^{n}\right)$ given by

$$
U_{0}(t) \vec{g}=\left(u_{0}(t, \cdot), \partial_{t} u_{0}(t, \cdot)\right), \quad \vec{g}=\left(g_{0}, g_{1}\right) \in \mathscr{H}_{D}\left(\boldsymbol{R}^{n}\right)
$$

with $u_{0}$ being the solution to the Cauchy problem

$$
\begin{aligned}
& \left(\partial_{t}^{2}-\Delta\right) u_{0}(t, x)=0, \quad(t, x) \in(0, \infty) \times \boldsymbol{R}^{n}, \\
& u_{0}(0, x)=g_{0}(x),\left(\partial_{t} u_{0}\right)(0, x)=g_{1}(x), \quad x \in \boldsymbol{R}^{n} .
\end{aligned}
$$

It is also known that the local energy of $U(t) \vec{f}$ decays to zero as $t$ tends to infinity. Namely, for any $R>0$ and any $\vec{f} \in \mathscr{H}_{D}(\Omega)$, we have

$$
\lim _{t \rightarrow \infty} \int_{\{x \in \Omega ;|x|<R\}}\left\{\left|\partial_{t} u(t, x)\right|^{2}+|\nabla u(t, x)|^{2}\right\} d x=0
$$

where $u$ is the solution to (1.1)-(1.3).

In view of these facts, we see that the main part of the perturbed wave $U(t) \vec{f}$ escapes from any ball with a fixed radius as $t \rightarrow \infty$ and that it approaches some unperturbed wave $U_{0}(t) \vec{f}_{+}$in the sense of the energy. However, to our knowledge, the rate of the convergence in (1.4) is not found explicitly in the literature. Therefore, it is natural to ask at which rate the perturbed wave tends to an unperturbed wave. In addition, we are interested in the regularity and decay properties of the scattering data $\vec{f}_{+}$. Namely, we wish to know whether the scattering data becomes smoother and decays faster at the spatial infinity or not, if the initial data is smooth and compactly supported. This consideration might be useful for the application to the nonlinear wave equation in an exterior domain. For instance, we are able to obtain a precise lower bound of the lifespan in our forthcoming paper [13].

Here we introduce notation in order to state our main result. Let $m$ be a nonnegative integer and $Y$ be an open set in $\boldsymbol{R}^{n}$. We set $\mathscr{H}^{m}(Y)=$ $H^{m+1}(Y) \times H^{m}(Y)$ and $\|\vec{f}\|_{\mathscr{H}^{m}(Y)}=\left\|f_{0}\right\|_{H^{m+1}(Y)}+\left\|f_{1}\right\|_{H^{m}(Y)}$ for $\vec{f}=\left(f_{0}, f_{1}\right)$ $\in \mathscr{H}^{m}(Y)$. Similarly, we put $\mathscr{W}^{m, \infty}(Y)=W^{m+1, \infty}(Y) \times W^{m, \infty}(Y)$ and $\|\vec{f}\|_{\mathscr{W}^{m, \infty}(Y)}=\left\|f_{0}\right\|_{W^{m+1, \infty}(Y)}+\left\|f_{1}\right\|_{W^{m, \infty}(Y)}$ for $\vec{f} \in \mathscr{W}^{m, \infty}(Y)$. Here $H^{m}(Y)$ (resp. $\left.W^{m, \infty}(Y)\right)$ stands for the Sobolev space based on $L^{2}(Y)$ (resp. $L^{\infty}(Y)$ ). In addition, we denote by $X^{m}(\Omega)$ the set of all $\vec{f}=\left(f_{0}, f_{1}\right) \in \mathscr{H}^{m}(\Omega)$ satisfying 
the compatibility condition of the $m$-th order for the problem (1.1)-(1.3), that is to say, $f_{j}=0$ on $\partial \Omega$ for any $j=0, \ldots, m$, where we have set

$$
f_{j}(x) \equiv \Delta f_{j-2}(x) \quad \text { for } x \in \bar{\Omega} \text { and } j \geq 2 .
$$

Besides, we put $\mathscr{H}^{\infty}(Y)=\bigcap_{m=0}^{\infty} \mathscr{H}^{m}(Y)$ and $X^{\infty}(\Omega)=\bigcap_{m=0}^{\infty} X^{m}(\Omega)$.

We will use the notation $\partial_{j}=\partial_{x_{j}}$ for $1 \leq j \leq n$, and $\partial_{x}^{\alpha}=\partial_{1}^{\alpha_{1}} \ldots \partial_{n}^{\alpha_{n}}$ for a multi-index $\alpha=\left(\alpha_{1}, \ldots, \alpha_{n}\right)$. We set

$$
Z=\left(Z_{0}, Z_{1}, \ldots, Z_{N}\right)=\left(\partial_{t}, \partial_{1}, \ldots, \partial_{n},\left(O_{i j}\right)_{1 \leq i<j \leq n}\right)
$$

with $\quad N=n(n+1) / 2$, and $Z^{\beta}=Z_{0}^{\beta_{0}} Z_{1}^{\beta_{1}} \ldots Z_{N}^{\beta_{N}}$ for a multi-index $\beta=$ $\left(\beta_{0}, \beta_{1}, \ldots, \beta_{N}\right)$, where $O_{i j}$ for $1 \leq i, j \leq n$ is given by $O_{i j}=x_{i} \partial_{j}-x_{j} \partial_{i}$.

For $r>0$ and $y \in \boldsymbol{R}^{n}, B_{r}(y)$ stands for an open ball of radius $r$ centered at $y$. We write $B_{r}$ for $B_{r}(0)$. Besides, we set $\Omega_{r}=\Omega \cap B_{r}$.

In what follows, for a constant $C$, when we write $C=C\left(p_{1}, \ldots, p_{m}\right)$ with $p_{1}, \ldots, p_{m}$ being some given constants or functions, it means that $C$ is a constant depending only on $p_{1}, \ldots, p_{m}$ if the space dimension $n$ and the obstacle $\mathcal{O}$ are fixed (thus $C$ may depend also on $n$ and $\mathcal{O}$ actually).

Our main result reads as follows.

Theorem 1.1. Let the space dimension $n$ be odd, and $n \geq 3$. Assume that $\mathcal{O}$ is non-trapping, and $\overline{\mathcal{O}} \subset B_{1}$. Let $a(>1)$ be a fixed number. Then for any $\vec{f} \in X^{\infty}(\Omega)$ with supp $\vec{f} \subset \overline{\Omega_{a}}$, there exists uniquely $\vec{f}_{+} \in \mathscr{H}^{\infty}\left(\boldsymbol{R}^{n}\right)$ satisfying (1.4). Moreover, there exists a positive constant $\mu=\mu(a)$ having the following property: For any nonnegative integer $k$, there exists a positive constant $C=$ $C(k, a)$ such that

$$
\begin{aligned}
& \left\|\exp (\mu\langle\cdot\rangle)\left(U(t) \vec{f}-\left.\left(U_{0}(t) \vec{f}_{+}\right)\right|_{\Omega}\right)\right\|_{\mathscr{H}^{k}(\Omega)} \\
& \leq C \exp (-\mu t)\|\vec{f}\|_{\mathscr{H}^{k}(\Omega)} \quad \text { for } t \geq 0 \\
& \left\|\exp (2 \mu\langle\cdot\rangle) \vec{f}_{+}\right\|_{\mathscr{W}^{k, \infty}\left(\boldsymbol{R}^{n}\right)} \leq C\|\vec{f}\|_{\mathscr{H}^{k+[n / 2]+1}(\Omega)},
\end{aligned}
$$

where $\langle x\rangle=\sqrt{1+|x|^{2}}$ for $x \in \boldsymbol{R}^{n}$, and $[n / 2]$ denotes the largest integer not exceeding $n / 2$.

Theorem 1.1 will be proved in Section 3. Our proof of Theorem 1.1 relies on the exponential decay of the local energy (see Lemma 2.5 below), and this is the reason why $n(\geq 3)$ is assumed to be odd and the obstacle $\mathcal{O}$ to be nontrapping. For the notion of the non-trapping obstacle, we refer to Melrose [10] for instance (see also Shibata-Tsutsumi [11, 12]). For example, star-shaped obstacles are known to be non-trapping.

Note that (1.10) implies that each component of $\vec{f}_{+}$belongs to the Schwartz class $\mathscr{S}$, the class of rapidly decreasing functions. 
Now we turn our attention to the asymptotic pointwise behavior of the perturbed wave $U(t) \vec{f}$. To describe the result, we define the Friedlander radiation field $\mathscr{F}_{0}[\vec{g}]$ by

$$
\widetilde{F}_{0}[\vec{g}](s, \eta)=\frac{1}{2(2 \pi)^{(n-1) / 2}} \sum_{j=0}^{1}\left(-\partial_{s}\right)^{(n-1) / 2-j} \mathscr{R}\left[g_{j}\right](s, \eta)
$$

for $\vec{g}=\left(g_{0}, g_{1}\right) \in\left(\mathscr{S}\left(\boldsymbol{R}^{n}\right)\right)^{2}$. Here $\mathscr{R}[\varphi]$ denotes the Radon transform of $\varphi=\varphi(x)$, that is

$$
\mathscr{R}[\varphi](s, \eta)=\int_{y \cdot \eta=s} \varphi(y) d S_{y},
$$

where $d S_{y}$ denotes the area element on the hyperplane $\{y ; y \cdot \eta=s\}$. The radiation field $\mathscr{F}_{0}[\vec{g}]$ is introduced to describe the main part of the unperturbed wave $U_{0}(t) \vec{g}$ for $\vec{g} \in\left(C_{0}^{\infty}\left(\boldsymbol{R}^{3}\right)\right)^{2}$ in Friedlander [2]. The Friedlander radiation field is also closely connected to the translation representation for the wave equation (see Lax-Phillips [9], Friedlander [5] for instance). By Theorem 1.1 and the Sobolev imbedding theorem, we immediately see that the main part of $U(t) \vec{f}$ can be described by $\mathscr{F}_{0}\left[\vec{f}_{+}\right]$, and we would like to investigate its convergence rate. Our result is the following.

Theorem 1.2. Let the assumptions of Theorem 1.1 hold. Then for any $\vec{f} \in X^{\infty}(\Omega)$ with supp $\vec{f} \subset \overline{\Omega_{a}}$, there exists $\vec{f}_{+} \in\left(\mathscr{S}\left(\boldsymbol{R}^{n}\right)\right)^{2}$ satisfying the following property: For any nonnegative integer $k$, there exists a positive constant $C=$ $C(k, a, \vec{f})$ such that, writing $x=r \omega$ with $r=|x|$ and $\omega=\left(\omega_{1}, \omega_{2}, \ldots, \omega_{n}\right) \in S^{n-1}$, we have

$$
\begin{aligned}
& \sum_{|\alpha| \leq k}\left|Z^{\alpha}\left\{u(t, x)-r^{-(n-1) / 2} \mathscr{F}_{0}\left[\vec{f}_{+}\right](r-t, \omega)\right\}\right| \\
& \leq C(1+t+r)^{-(n+1) / 2} \exp \left(-\frac{\mu}{2}|r-t|\right), \\
& \sum_{|\alpha| \leq k}\left|Z^{\alpha}\left\{\partial_{t} u(t, x)-(-1) r^{-(n-1) / 2}\left(\partial_{s} \mathscr{F}_{0}\left[\vec{f}_{+}\right]\right)(r-t, \omega)\right\}\right| \\
& \quad+\sum_{|\alpha| \leq k} \sum_{j=1}^{n}\left|Z^{\alpha}\left\{\partial_{j} u(t, x)-\omega_{j} r^{-(n-1) / 2}\left(\partial_{s} \mathscr{F}_{0}\left[\vec{f}_{+}\right]\right)(r-t, \omega)\right\}\right| \\
& \leq C(1+t+r)^{-(n+1) / 2} \exp \left(-\frac{\mu}{2}|r-t|\right)
\end{aligned}
$$

for $(t, x) \in(0, \infty) \times \Omega$ satisfying $r \geq t / 2 \geq 1$, where $u(t, x)$ is the solution to (1.1)-(1.3), and $\mu=\mu(a)$ is the positive constant from Theorem 1.1. 
The proof of Theorem 1.2 will be given in Section 5, after obtaining the detailed convergence rate for the Cauchy problem in Section 4 (see Proposition 4.1).

We underline that the decaying factor $\exp (-\mu|r-t| / 2)$ in the above estimates is quite meaningful even if the initial data is compactly supported, say supp $\vec{f} \subset \overline{\Omega_{a}}$, unlike the case of the Cauchy problem. In fact, the solution $u(t, x)$ for the mixed problem is not expected to vanish for $r-t \leq-a$ in general, because of the presence of the obstacle, while it is identically zero for $r-t \geq a$ and $t \geq 0$, in view of the domain of dependence (see Lemma 2.2 below). Accordingly, the radiation field $\mathscr{F}_{0}\left[\vec{f}_{+}\right](s, \omega)$ for the solution to the mixed problem is not supposed to be zero for $s \leq-a$ and $\omega \in S^{n-1}$ in general, while it vanishes for $s \geq a$ and $\omega \in S^{n-1}$ due to (1.12). In contrast to this, if there is no obstacle, it is known that the radiation field for compactly supported data vanishes not only for $s \geq a$, but also for $s \leq-a$ (this property is closely connected to the Huygens principle; see Lemma 2.3 below). In conclusion, it is essential to extract the decay factor with respect to $|r-t|$, in order to describe the behavior for the mixed problem in the region $r-t \leq-a$.

\section{Preliminaries}

Let $Y$ be an open subset of $\boldsymbol{R}^{n}$, and $\Omega$ be as in the previous section. For the notational convenience, we put

$$
\begin{aligned}
& \mathscr{H}_{a}^{\infty}(Y)=\left\{\vec{f}=\left(f_{0}, f_{1}\right) \in \mathscr{H}^{\infty}(Y) ; \operatorname{supp} \vec{f} \subset \overline{Y \cap B_{a}}\right\}, \\
& X_{a}^{\infty}(\Omega)=\left\{\vec{f}=\left(f_{0}, f_{1}\right) \in X^{\infty}(\Omega) ; \operatorname{supp} \vec{f} \subset \overline{\Omega_{a}}\right\}
\end{aligned}
$$

for $a>0$.

$U_{0}(t)$ and $U(t)$ are unitary in $\mathscr{H}_{D}\left(\boldsymbol{R}^{n}\right)$ and $\mathscr{H}_{D}(\Omega)$, respectively, and the energy inequalities for higher derivatives are also available: For a nonnegative integer $k$, there exists a positive constant $C_{k}$ such that

$$
\sum_{|\alpha| \leq k}\left\|\partial_{x}^{\alpha} U_{0}(t) \vec{g}\right\|_{\mathscr{H}_{D}\left(\boldsymbol{R}^{n}\right)} \leq C_{k} \sum_{|\alpha| \leq k}\left\|\partial_{x}^{\alpha} \vec{g}\right\|_{\mathscr{H}_{D}\left(\boldsymbol{R}^{n}\right)}, \quad t \in \boldsymbol{R}
$$

for any $\vec{g} \in \mathscr{H}^{k}\left(\boldsymbol{R}^{n}\right)$, and

$$
\sum_{|\alpha| \leq k}\left\|\partial_{x}^{\alpha} U(t) \vec{f}\right\|_{\mathscr{H}_{D}(\Omega)} \leq C_{k} \sum_{|\alpha| \leq k}\left\|\partial_{x}^{\alpha} \vec{f}\right\|_{\mathscr{H}_{D}(\Omega)}, \quad t \in \boldsymbol{R}
$$

for any $\vec{f} \in X^{k}(\Omega)$, where $\partial_{x}=\left(\partial_{1}, \ldots, \partial_{n}\right)$ and $\alpha$ is a multi-index. Combining (2.3) or (2.4) with the elementary inequality

$$
\|h(t)\|_{L^{2}(Y)} \leq\left\|h\left(t_{0}\right)\right\|_{L^{2}(Y)}+\int_{t_{0}}^{t}\left\|\partial_{t} h(\tau)\right\|_{L^{2}(Y)} d \tau, \quad t \geq t_{0},
$$


which is valid for any smooth function $h$ and any open subset $Y$ of $\boldsymbol{R}^{n}$, we obtain the following estimates.

Lemma 2.1. Let $k$ be a nonnegative integer. Then we have

$$
\left\|U_{0}(t) \vec{g}\right\|_{\mathscr{H}^{k}\left(\boldsymbol{R}^{n}\right)} \leq C_{k}(1+|t|)\|\vec{g}\|_{\mathscr{H}^{k}\left(\boldsymbol{R}^{n}\right)}, \quad t \in \boldsymbol{R}
$$

for any $\vec{g} \in \mathscr{H}^{k}\left(\boldsymbol{R}^{n}\right)$. We also have

$$
\|U(t) \vec{f}\|_{\mathscr{H}^{k}(\Omega)} \leq C_{k}(1+|t|)\|\vec{f}\|_{\mathscr{H}^{k}(\Omega)}, \quad t \in \boldsymbol{R}
$$

for any $\vec{f} \in X^{k}(\Omega)$.

The following property is well known (see [9, Chapter V] for instance).

Lemma 2.2 (Domain of dependence). Let $n$ be a positive integer. Let $\tau, t_{0} \in \boldsymbol{R}$ with $\tau<t_{0}$, and let $x_{0} \in \boldsymbol{R}^{n}$. We define

$$
\Lambda\left(t_{0}, x_{0}, \tau\right)=\left\{(t, x) \in\left(\tau, t_{0}\right) \times \boldsymbol{R}^{n} ;\left|x-x_{0}\right|<t_{0}-t\right\} .
$$

Suppose that $\psi=\psi(t, x)$ satisfies

$$
\left(\partial_{t}^{2}-\Delta\right) \psi(t, x)=0, \quad(t, x) \in \Lambda\left(t_{0}, x_{0}, \tau\right) .
$$

Then we have

$$
\|\partial \psi(t)\|_{L^{2}\left(B_{t_{0}-t}\left(x_{0}\right)\right)} \leq\|\partial \psi(\tau)\|_{L^{2}\left(B_{t_{0}-\tau}\left(x_{0}\right)\right)}, \quad t \in\left(\tau, t_{0}\right),
$$

where $\partial \psi=\left(\partial_{t} \psi, \nabla \psi\right)$. As a consequence, if we also assume

$$
\psi(\tau, x)=\left(\partial_{t} \psi\right)(\tau, x)=0, \quad x \in B_{t_{0}-\tau}\left(x_{0}\right),
$$

then we have $\psi(t, x)=0$ for any $(t, x) \in \Lambda\left(t_{0}, x_{0}, \tau\right)$.

The above assertions are also valid if we replace $\Lambda\left(t_{0}, x_{0}, \tau\right)$ by

$$
\Lambda^{*}\left(t_{0}, x_{0}, \tau\right)=\left\{(t, x) \in\left(2 \tau-t_{0}, \tau\right) \times \boldsymbol{R}^{n} ;\left|x-x_{0}\right|<t+t_{0}-2 \tau\right\},
$$

and (2.8) by

$$
\|\partial \psi(t)\|_{L^{2}\left(B_{t+t_{0}-2 \tau}\left(x_{0}\right)\right)} \leq\|\partial \psi(\tau)\|_{L^{2}\left(B_{t_{0}-\tau}\left(x_{0}\right)\right)}, \quad t \in\left(2 \tau-t_{0}, \tau\right) .
$$

From the lemma above, we see that $\vec{f} \in X_{a}^{\infty}(\Omega)$ (resp. $\vec{g} \in \mathscr{H}_{a}^{\infty}\left(\boldsymbol{R}^{n}\right)$ ) implies $\operatorname{supp}(U(t) \vec{f}) \subset \overline{\Omega_{|t|+a}}\left(\right.$ resp. $\left.\operatorname{supp}\left(U_{0}(t) \vec{g}\right) \subset \overline{B_{|t|+a}}\right)$.

In odd space dimensions, we have a stronger result.

Lemma 2.3 (The Huygens principle). Let $n$ be an odd integer with $n \geq 3$. Then $\vec{g} \in \mathscr{H}_{a}^{\infty}\left(\boldsymbol{R}^{n}\right)$ implies

$$
\operatorname{supp}\left(U_{0}(t) \vec{g}\right) \subset\left\{x \in \boldsymbol{R}^{n} ;|t|-a \leq|x| \leq|t|+a\right\}, \quad t \in \boldsymbol{R} .
$$


Lemma 2.3 follows immediately from the explicit expression of $U_{0}(t) \vec{g}$ (see (4.30) below).

For the later usage, we mention an immediate consequence of Lemma 2.3 for inhomogeneous wave equations:

Corollary 2.4. Let $\varphi$ be a solution to

$$
\begin{cases}\left(\partial_{t}^{2}-\Delta\right) \varphi(t, x)=F(t, x), & (t, x) \in(0, \infty) \times \boldsymbol{R}^{n}, \\ \left(\varphi(0, x),\left(\partial_{t} \varphi\right)(0, x)\right)=\vec{g}(x), & x \in \boldsymbol{R}^{n},\end{cases}
$$

where $\vec{g} \in \mathscr{H}^{\infty}\left(\boldsymbol{R}^{n}\right)$, and $F \in C^{\infty}\left([0, \infty) \times \boldsymbol{R}^{n}\right)$. Suppose that

$$
\operatorname{supp} \vec{g} \subset \overline{B_{a}}, \quad \text { and } \quad \operatorname{supp} F \subset\left[0, T_{0}\right] \times \overline{B_{b}}
$$

with some positive constants $a, b$, and $T_{0}$. Then for $t \geq T_{0}$ we have

$$
\operatorname{supp} \varphi(t, \cdot) \subset D\left(\min \left\{t-a,\left(t-T_{0}\right)-b\right\}, \max \{t+a, t+b\}\right),
$$

where $D\left(r_{1}, r_{2}\right)=\left\{x \in \boldsymbol{R}^{n} ; r_{1} \leq|x| \leq r_{2}\right\}$ for $0 \leq r_{1}<r_{2}$.

Proof. By the Duhamel principle, $\left(\varphi, \partial_{t} \varphi\right)$ can be written as

$$
\left(\varphi(t, x), \partial_{t} \varphi(t, x)\right)=\left(U_{0}(t) \vec{g}\right)(x)+\int_{0}^{t}\left(U_{0}(t-\tau) \vec{F}(\tau, \cdot)\right)(x) d \tau,
$$

where $\vec{F}(t, x)=(0, F(t, x))$. For $t \geq T_{0}$, it follows from (2.9), (2.10), and Lemma 2.3 that

$$
\operatorname{supp} \varphi(t, \cdot) \subset D(t-a, t+a) \cup \bigcup_{\tau \in\left[0, T_{0}\right]} D((t-\tau)-b,(t-\tau)+b),
$$

which implies the desired result immediately.

Next we introduce the local energy decay of the perturbed wave at exponential rate (for the proof, see for instance Melrose [10]; see also ShibataTsutsumi [11]).

Lemma 2.5. Let $n$ be odd and $n \geq 3$. Assume that $\mathcal{O}$ is non-trapping, and $\overline{\mathcal{O}} \subset B_{1}$. Suppose that $a, b>1$, and $k$ is a nonnegative integer. Then there exist two positive constants $C=C(k, a, b)$ and $\sigma=\sigma(a, b)$ such that for any $\vec{f} \in X_{a}^{\infty}(\Omega)$ we have

$$
\|U(t) \vec{f}\|_{\mathscr{H}^{k}\left(\Omega_{b}\right)} \leq C \exp (-\sigma t)\|\vec{f}\|_{\mathscr{H}^{k}(\Omega)} \quad \text { for } t \geq 0 .
$$

The following lemma, motivated by the arguments in Ikawa [7], tells us that the perturbed wave can be decomposed into the unperturbed wave and the correction term. The former is the main part of the perturbed wave, while the latter takes care of the effect from the boundary and its size can be small compared with the initial energy. This lemma is crucial for proving Theorem 1.1. 
Lemma 2.6. Let $n$, $\mathcal{O}$, and $a$ be as in Theorem 1.1. Then, for any $\vec{f} \in X_{a}^{\infty}(\Omega)$ and $T(\geq a+2)$, there exist $\vec{g}_{1} \in \mathscr{H}_{T+a}^{\infty}\left(\boldsymbol{R}^{n}\right)$ and $\vec{f}_{1} \in X_{3}^{\infty}(\Omega)$ satisfying

$$
\begin{aligned}
& U(t) \vec{f}=\left.\left(U_{0}(t-T) \vec{g}_{1}\right)\right|_{\Omega}+U(t-T) \vec{f}_{1}, \quad t \geq T, \\
& \operatorname{supp} U_{0}(t-T) \vec{g}_{1} \subset D(t-T+1, t+a), \quad t \geq T, \\
& \left\|\vec{g}_{1}\right\|_{\mathscr{H}^{k}\left(\boldsymbol{R}^{n}\right)} \leq C_{0}(1+T)\|\vec{f}\|_{\mathscr{H}^{k}(\Omega)}, \\
& \left\|\vec{f}_{1}\right\|_{\mathscr{H}^{k}(\Omega)} \leq C_{0} \exp (-\sigma T)\|\vec{f}\|_{\mathscr{H}^{k}(\Omega)}
\end{aligned}
$$

for any nonnegative integer $k$ with some positive constants $C_{0}=C_{0}(k, a)$ and $\sigma=\sigma(a)$. Here $D\left(r_{1}, r_{2}\right)$ is defined in Corollary 2.4.

Remark. By (2.13), we see that $U_{0}(t-T) \vec{g}_{1}$ vanishes on $\overline{\mathcal{O}}\left(\subset B_{1}\right)$ for $t \geq T$. Thus the uniqueness of the solution to the mixed problem (1.1)-(1.3) implies

$$
\left.\left(U_{0}(t-T) \vec{g}_{1}\right)\right|_{\Omega}=U(t-T)\left(\left.\vec{g}_{1}\right|_{\Omega}\right), \quad t \geq T .
$$

Proof. In this proof, various positive constants depending only on $k$ will be indicated by the same letter $C_{k}$. Let $\vec{f} \in X_{a}^{\infty}(\Omega)$ be a given function.

Step 1: Construction of $\vec{g}_{1}$ and proof of (2.14). Let $\chi_{1}=\chi_{1}(x)$ be a smooth and nonnegative function satisfying $\chi_{1}(x)=1$ for $|x| \geq 1$, and $\chi_{1}(x)=0$ for $x \in \overline{\mathcal{O}}$. For a function $F$ on $\Omega, \mathscr{E}[F]$ denotes the extension of $F$ by 0 outside $\Omega$. We put

$$
\vec{\psi}:=\chi_{1} \mathscr{E}[U(T-2) \vec{f}] \quad \text { in } \boldsymbol{R}^{n}
$$

and we define

$$
\vec{g}_{1}:=U_{0}(2) \vec{\psi} \quad \text { in } \boldsymbol{R}^{n}
$$

Then we have $\vec{g}_{1} \in \mathscr{H}_{T+a}^{\infty}\left(\boldsymbol{R}^{n}\right)$. Indeed, since $\vec{f} \in X_{a}^{\infty}(\Omega)$, Lemma 2.2 implies $U(T-2) \vec{f} \in X_{T-2+a}^{\infty}(\Omega)$; consequently we get $\vec{\psi} \in \mathscr{H}_{T-2+a}^{\infty}\left(\boldsymbol{R}^{n}\right)$, which implies $\vec{g}_{1} \in \mathscr{H}_{T+a}^{\infty}\left(\boldsymbol{R}^{n}\right)$ by Lemma 2.2 again.

Next we show (2.14). By (2.17) and (2.7), we obtain

$$
\|\vec{\psi}\|_{\mathscr{H}^{k}\left(\boldsymbol{R}^{n}\right)} \leq C_{k}\|U(T-2) \vec{f}\|_{\mathscr{H}^{k}(\Omega)} \leq C_{k}(T-1)\|\vec{f}\|_{\mathscr{H}^{k}(\Omega)} .
$$

Therefore, from (2.18) and (2.6), we obtain

$$
\left\|\vec{g}_{1}\right\|_{\mathscr{H}^{k}\left(\boldsymbol{R}^{n}\right)} \leq C_{k}\|\vec{\psi}\|_{\mathscr{H}^{k}\left(\boldsymbol{R}^{n}\right)} \leq C_{k}(T-1)\|\vec{f}\|_{\mathscr{H}^{k}(\Omega)},
$$

which implies (2.14). 
Step 2: Proof of (2.13). We prove (2.13) by applying Corollary 2.4 in the following way: We define a function $z$ on $[0, \infty) \times \boldsymbol{R}^{n}$ by

$$
z(t, x)= \begin{cases}u(t, x) & \text { for }(t, x) \in[0, T-2] \times \Omega, \\ 0 & \text { for }(t, x) \in[0, T-2] \times \overline{\mathcal{O}}, \\ v(t, x) & \text { for }(t, x) \in(T-2, \infty) \times \boldsymbol{R}^{n}\end{cases}
$$

where $u$ and $v$ are given by

$$
\begin{aligned}
\left(u(t, x),\left(\partial_{t} u\right)(t, x)\right) & :=(U(t) \vec{f})(x), \quad t \geq 0, x \in \Omega, \\
\left(v(t, x),\left(\partial_{t} v\right)(t, x)\right) & :=\left(U_{0}(t-T) \vec{g}_{1}\right)(x) \\
& =\left(U_{0}(t-T+2) \vec{\psi}\right)(x), \quad t \geq T-2, x \in \boldsymbol{R}^{n} .
\end{aligned}
$$

Noting that we have

$$
\left(u(t, x),\left(\partial_{t} u\right)(t, x)\right)=\vec{\psi}(x)=\left(v(t, x),\left(\partial_{t} v\right)(t, x)\right)
$$

for $t=T-2$ and $|x| \geq 1$ (cf. (2.17)), we see that $z$ is smooth in $[0, \infty) \times$ $\left(\boldsymbol{R}^{n} \backslash B_{1}\right)$, and $\left(\partial_{t}^{2}-\Delta\right) z=0$ in $[0, \infty) \times\left(\boldsymbol{R}^{n} \backslash B_{1}\right)$. We cut off $z$ to make it smooth in $[0, \infty) \times \boldsymbol{R}^{n}$ : For $\varepsilon>0$, let $\chi_{2, \varepsilon}$ be a smooth function in $[0, \infty) \times \boldsymbol{R}^{n}$ such that

$$
\chi_{2, \varepsilon}(t, x)= \begin{cases}1 & \text { for }|x| \geq 1+\varepsilon \text { or } t \geq T-2+\varepsilon, \\ 0 & \text { for }(t, x) \in[0, T-2] \times B_{1} .\end{cases}
$$

For $(t, x) \in[0, \infty) \times \boldsymbol{R}^{n}$ we define

$$
z_{\varepsilon}(t, x)=\chi_{2, \varepsilon}(t, x) z(t, x)
$$

Note that we have

$$
U_{0}(t-T) \vec{g}_{1}=\left(z_{\varepsilon}(t), \partial_{t} z_{\varepsilon}(t)\right), \quad t \geq T-2+\varepsilon
$$

by definition. Let $\varepsilon$ be a positive and small constant satisfying $1+\varepsilon \leq a$. We are going to show

$$
\operatorname{supp} z_{\varepsilon}(t, \cdot) \subset D(t-T+1-2 \varepsilon, t+a)
$$

for $t \geq T-2+\varepsilon$. Note that (2.13) follows from (2.20) and (2.21): Indeed it follows from (2.20) and (2.21) that

$$
\text { supp } U_{0}(t-T) \vec{g}_{1} \subset D(t-T+1-2 \varepsilon, t+a)
$$

for $t \geq T(>T-2+\varepsilon)$, provided that $\varepsilon$ satisfies $0<\varepsilon \leq a-1$ and is small; from (2.22) we obtain (2.13) because we have

$$
\bigcap_{0<\varepsilon \leq \varepsilon_{0}} D(t-T+1-2 \varepsilon, t+a)=D(t-T+1, t+a)
$$


for any $\varepsilon_{0}>0$. We start the proof of (2.21). In view of Corollary 2.4, our task is to investigate the support of $\left(z_{\varepsilon}(0), \partial_{t} z_{\varepsilon}(0)\right)$ and $\left(\partial_{t}^{2}-\Delta\right) z_{\varepsilon}$. Since $\vec{f} \in X_{a}^{\infty}(\Omega)$, it is easy to see

$$
\operatorname{supp} z_{\varepsilon}(0, \cdot) \cup \operatorname{supp} \partial_{t} z_{\varepsilon}(0, \cdot) \subset \overline{B_{a}} .
$$

Since $\left(\partial_{t}^{2}-\Delta\right) z(t, x)=0$ for $(t, x) \in \operatorname{supp} \chi_{2, \varepsilon}$, we get

$$
\left(\partial_{t}^{2}-\Delta\right) z_{\varepsilon}=\left(\partial_{t}^{2} \chi_{2, \varepsilon}-\Delta \chi_{2, \varepsilon}\right) z+2\left\{\left(\partial_{t} \chi_{2, \varepsilon}\right)\left(\partial_{t} z\right)-\nabla \chi_{2, \varepsilon} \cdot \nabla z\right\} .
$$

Hence we obtain

$$
\operatorname{supp}\left(\partial_{t}^{2}-\Delta\right) z_{\varepsilon} \subset[0, T-2+\varepsilon] \times \overline{B_{1+\varepsilon}},
$$

because supp $\partial_{t, x}^{\alpha} \chi_{2, \varepsilon} \subset[0, T-2+\varepsilon] \times \overline{B_{1+\varepsilon}}$ for $|\alpha|=1,2$. By (2.23) and (2.24) we can apply Corollary 2.4 (with $\varphi=z_{\varepsilon}, \vec{g}=\left(z_{\varepsilon}(0), \partial_{t} z_{\varepsilon}(0)\right), F=\left(\partial_{t}^{2}-\Delta\right) z_{\varepsilon}$, $T_{0}=T-2+\varepsilon$, and $\left.b=1+\varepsilon\right)$ to obtain (2.21) for $t \geq T_{0}$ (note that we have $\min \{t-a, t-(T-2+\varepsilon)-(1+\varepsilon)\}=t-T+1-2 \varepsilon$ and $\max \{t+a, t+(1+\varepsilon)\}$ $=t+a$ because $1+\varepsilon \leq a \leq T-2)$.

Step 3: Construction of $f_{1}$, and proof of (2.12) and (2.15). We define

$$
\vec{f}_{1}:=U(T) \vec{f}-\left.\vec{g}_{1}\right|_{\Omega} \quad \text { in } \Omega .
$$

Since (2.13) implies (2.16), we get

$$
\begin{aligned}
& \left.\left(U_{0}(t-T) \vec{g}_{1}\right)\right|_{\Omega}+U(t-T) \vec{f}_{1} \\
& \quad=U(t-T)\left(\left.\vec{g}_{1}\right|_{\Omega}+\vec{f}_{1}\right)=U(t-T) U(T) \vec{f}=U(t) \vec{f}
\end{aligned}
$$

for $t \geq T$, which shows (2.12).

Next we will show that $\vec{f}_{1} \in X_{3}^{\infty}(\Omega)$. From (2.13), we see that $\vec{g}_{1}$ vanishes in an open set containing $\partial \Omega$. Thus the compatibility condition of the infinite order is satisfied for $\left.\vec{g}_{1}\right|_{\Omega}$, and we find

$$
\left.\vec{g}_{1}\right|_{\Omega} \in X_{T+a}^{\infty}(\Omega) .
$$

Since $U(T) \vec{f} \in X^{\infty}(\Omega)$, from (2.25) and (2.26) we get $\vec{f}_{1} \in X^{\infty}(\Omega)$. Hence it remains to prove

$$
\text { supp } \vec{f}_{1} \subset \overline{\Omega_{3}}
$$

in order to show $\vec{f}_{1} \in X_{3}^{\infty}(\Omega)$. For the proof of $(2.27)$, we put

$$
\left(w(t, x),\left(\partial_{t} w\right)(t, x)\right):=(U(t) \vec{f})(x)-\left(U_{0}(t-T+2) \vec{\psi}\right)(x)
$$

for $(t, x) \in[T-2, \infty) \times \Omega$. Then we have

$$
\square w(t, x)=0, \quad(t, x) \in(T-2, \infty) \times \Omega .
$$


From (2.17) we also get

(2.29) $\quad\left(w(T-2, x),\left(\partial_{t} w\right)(T-2, x)\right)=(U(T-2) \vec{f})(x)-\vec{\psi}(x)=(0,0)$

for $x \in \boldsymbol{R}^{n} \backslash B_{1}$. If $t \geq T-2$ and $|x| \geq t-T+3$, then we have

$$
\Lambda(t, x, T-2) \subset[T-2, \infty) \times\left(\boldsymbol{R}^{n} \backslash B_{1}\right),
$$

where $\Lambda\left(t_{0}, x_{0}, \tau\right)$ is from Lemma 2.2. Hence (2.28), (2.29), and Lemma 2.2 imply

$$
w(t, x)=0 \quad \text { for }|x| \geq t-T+3, t \geq T-2,
$$

which shows $(2.27)$ because we have $\vec{f}_{1}=\left(w(T),\left(\partial_{t} w\right)(T)\right)$.

Finally we are going to prove (2.15). From (2.18), (2.25), and (2.27), we get

$$
\left\|\vec{f}_{1}\right\|_{\mathscr{H}^{k}(\Omega)} \leq\|U(T) \vec{f}\|_{\mathscr{H}^{k}\left(\Omega_{3}\right)}+\left\|U_{0}(2) \vec{\psi}\right\|_{\mathscr{H}^{k}\left(B_{3}\right)} .
$$

It follows from (2.8), (2.5) and (2.17) that

$$
\left\|U_{0}(2) \vec{\psi}\right\|_{\mathscr{H}^{k}\left(B_{3}\right)} \leq C_{k}\|\vec{\psi}\|_{\mathscr{H}^{k}\left(B_{5}\right)} \leq C_{k}\|U(T-2) \vec{f}\|_{\mathscr{H}^{k}\left(\Omega_{5}\right)} .
$$

Using Lemma 2.5, from (2.31) and (2.32) we obtain

$$
\begin{aligned}
\left\|\vec{f}_{1}\right\|_{\mathscr{H}^{k}(\Omega)} & \leq\|U(T) \vec{f}\|_{\mathscr{H}^{k}\left(\Omega_{3}\right)}+C_{k}\|U(T-2) \vec{f}\|_{\mathscr{H}^{k}\left(\Omega_{5}\right)} \\
& \leq C \exp (-\sigma T)\|\vec{f}\|_{\mathscr{H}^{k}(\Omega)},
\end{aligned}
$$

where $C=C(k, a)$ and $\sigma=\sigma(a)$ are positive constants. This completes the proof.

We conclude this section by showing the following lemma concerning the uniqueness of the scattering data.

Lemma 2.7. Let $\vec{f} \in X^{\infty}(\Omega)$. If $\vec{f}_{+} \in \mathscr{H}^{0}\left(\boldsymbol{R}^{n}\right)$ satisfies

$$
\lim _{t \rightarrow \infty}\left\|U(t) \vec{f}-\left.\left(U_{0}(t) \vec{f}_{+}\right)\right|_{\Omega}\right\|_{\mathscr{H}_{D}(\Omega)}=0
$$

then $\vec{f}_{+}$is determined uniquely.

Proof. Let $\vec{f}_{+} \in \mathscr{H}^{0}\left(\boldsymbol{R}^{n}\right)$ be as in the assertion and suppose that $\vec{g}_{+} \in$ $\mathscr{H}^{0}\left(\boldsymbol{R}^{n}\right)$ satisfies $\lim _{t \rightarrow \infty}\left\|U(t) \vec{f}-\left.\left(U_{0}(t) \vec{g}_{+}\right)\right|_{\Omega}\right\|_{\mathscr{H}_{D}(\Omega)}=0$. Then we have

$$
\lim _{t \rightarrow \infty}\left\|U_{0}(t)\left(\vec{f}_{+}-\vec{g}_{+}\right)\right\|_{\mathscr{H}_{D}(\Omega)}=0
$$


Therefore it suffices to show

$$
\lim _{t \rightarrow \infty}\left\|U_{0}(t)\left(\vec{f}_{+}-\vec{g}_{+}\right)\right\|_{\mathscr{H}_{D}\left(B_{1}\right)}=0 .
$$

In fact, once we obtain (2.35), then we see from (2.34) and the unitarity of $U_{0}(t)$ on $\mathscr{H}_{D}\left(\boldsymbol{R}^{n}\right)$ that $\vec{f}_{+}=\vec{g}_{+}$in $\mathscr{H}_{D}\left(\boldsymbol{R}^{n}\right)$. Moreover, the Hölder inequality and the Sobolev imbedding theorem imply that, for any $R>0$, there exists a positive constant $C_{R}$ such that we have

$$
\|v\|_{L^{2}\left(B_{R}\right)} \leq C_{R}\|v\|_{L^{2 n /(n-2)}\left(\boldsymbol{R}^{n}\right)} \leq C_{R}\|\nabla v\|_{L^{2}\left(\boldsymbol{R}^{n}\right)}
$$

for any $v \in \dot{H}^{1}\left(\boldsymbol{R}^{n}\right)$. We thus find $\vec{f}_{+}=\vec{g}_{+}$in $\mathscr{H}^{0}\left(\boldsymbol{R}^{n}\right)$.

Now we show (2.35). Since $\vec{f}_{+}, \vec{g}_{+} \in \mathscr{H}^{0}\left(\boldsymbol{R}^{n}\right)$, for any $\varepsilon>0$ there exists $\vec{h} \in\left(C_{0}^{\infty}\left(\boldsymbol{R}^{n}\right)\right)^{2}$ such that $\left\|\left(\vec{f}_{+}-\vec{g}_{+}\right)-\vec{h}\right\|_{\mathscr{H}_{D}\left(\boldsymbol{R}^{n}\right)}<\varepsilon$. Let supp $\vec{h} \subset \overline{B_{M}}$. Since the Huygens principle implies $U_{0}(t) \vec{h}=0$ for $|x| \leq 1$ and $t \geq M+1$, we obtain

$$
\begin{aligned}
\left\|U_{0}(t)\left(\vec{f}_{+}-\vec{g}_{+}\right)\right\|_{\mathscr{H}_{D}\left(B_{1}\right)} & =\left\|U_{0}(t)\left(\vec{f}_{+}-\vec{g}_{+}-\vec{h}\right)\right\|_{\mathscr{H}_{D}\left(B_{1}\right)} \\
& \leq\left\|\vec{f}_{+}-\vec{g}_{+}-\vec{h}\right\|_{\mathscr{H}_{D}\left(\boldsymbol{R}^{n}\right)}<\varepsilon
\end{aligned}
$$

for $t \geq M+1$, which leads to (2.35). This completes the proof.

\section{Proof of Theorem 1.1}

In this section we prove Theorem 1.1 by dividing the argument into four steps. We would like to construct $f_{+}$satisfying (1.9) and (1.10) for all $k$, but first we fix $k$ and construct $f_{+}$having the desired property for this fixed $k$. Then we will show that $f_{+}$is independent of $k$ in fact.

Step 1: Construction of $\vec{f}_{+}$with fixed $k$. We fix a nonnegative integer $k$ and set

$$
a_{*}=\max \{a, 3\}, \quad \mu=\sigma\left(a_{*}\right) / 4, \quad \text { and } \quad C_{1}=C_{0}\left(k, a_{*}\right),
$$

where $\sigma$ and $C_{0}$ are from Lemma 2.6. We choose $T\left(\geq a_{*}+2\right)$ to be so large that $C_{1} \exp (-\mu T) \leq 1$, and we put

$$
C_{2, T}:=C_{1}(1+T) \text {. }
$$

Then we see from Lemma 2.6 that for $\vec{f} \in X_{a}^{\infty}(\Omega)$, there exist $\vec{g}_{1} \in \mathscr{H}_{T+a_{*}}^{\infty}\left(\boldsymbol{R}^{n}\right)$ and $\vec{f}_{1} \in X_{3}^{\infty}(\Omega)$ satisfying (2.12),

$$
\left\|\vec{g}_{1}\right\|_{\mathscr{H}^{k}\left(\boldsymbol{R}^{n}\right)} \leq C_{1}(1+T)\|\vec{f}\|_{\mathscr{H}^{k}(\Omega)}=C_{2, T}\|\vec{f}\|_{\mathscr{H}^{k}(\Omega)}
$$

and

$$
\left\|\vec{f}_{1}\right\|_{\mathscr{H}^{k}(\Omega)} \leq C_{1} \exp (-4 \mu T)\|\vec{f}\|_{\mathscr{H}^{k}(\Omega)} \leq \exp (-3 \mu T)\|\vec{f}\|_{\mathscr{H}^{k}(\Omega)}
$$


We apply Lemma 2.6 to $\vec{f}_{1}$ again to find $\vec{g}_{2} \in \mathscr{H}_{T+a_{*}}^{\infty}\left(\boldsymbol{R}^{n}\right)$ and $\vec{f}_{2} \in X_{3}^{\infty}(\Omega)$ for which we have

$$
\begin{aligned}
& U(t-T) \vec{f}_{1}=\left.\left(U_{0}(t-2 T) \vec{g}_{2}\right)\right|_{\Omega}+U(t-2 T) \vec{f}_{2} \quad \text { for } t \geq 2 T \\
& \left\|\vec{g}_{2}\right\|_{\mathscr{H}^{k}\left(\boldsymbol{R}^{n}\right)} \leq C_{1}(1+T)\left\|\vec{f}_{1}\right\|_{\mathscr{H}^{k}(\Omega)} \leq C_{2, T} \exp (-3 \mu T)\|\vec{f}\|_{\mathscr{H}^{k}(\Omega)},
\end{aligned}
$$

and

$$
\left\|\vec{f}_{2}\right\|_{\mathscr{H}^{k}(\Omega)} \leq \exp (-3 \mu T)\left\|\vec{f}_{1}\right\|_{\mathscr{H}^{k}(\Omega)} \leq \exp (-6 \mu T)\|\vec{f}\|_{\mathscr{H}^{k}(\Omega)} .
$$

Repeating the same procedure, we can construct sequences $\left\{\vec{g}_{j}\right\}_{j=1}^{\infty} \subset \mathscr{H}_{T+a_{*}}^{\infty}\left(\boldsymbol{R}^{n}\right)$ and $\left\{\vec{f}_{j}\right\}_{j=1}^{\infty} \subset X_{3}^{\infty}(\Omega)$ in such a way that

$$
\begin{aligned}
& U(t-(j-1) T) \vec{f}_{j-1}=\left.\left(U_{0}(t-j T) \vec{g}_{j}\right)\right|_{\Omega}+U(t-j T) \vec{f}_{j}, \quad t \geq j T \\
& \left\|\vec{g}_{j}\right\|_{\mathscr{H}^{k}\left(\boldsymbol{R}^{n}\right)} \leq C_{2, T} \exp (-3 \mu(j-1) T)\|\vec{f}\|_{\mathscr{H}^{k}(\Omega)},
\end{aligned}
$$

and

$$
\left\|\vec{f}_{j}\right\|_{\mathscr{H}^{k}(\Omega)} \leq \exp (-3 \mu j T)\|\vec{f}\|_{\mathscr{H}^{k}(\Omega)}
$$

for $j \geq 1$, where we have put $\vec{f}_{0}=\vec{f}$.

Now we define

$$
\vec{f}_{+}=\sum_{j=1}^{\infty} U_{0}(-j T) \vec{g}_{j}
$$

Then it belongs to $\mathscr{H}^{\infty}\left(\boldsymbol{R}^{n}\right)$. In fact, (2.6) and (3.2) lead to

$$
\begin{aligned}
\left\|U_{0}(-j T) \vec{g}_{j}\right\|_{\mathscr{H}^{k}\left(\boldsymbol{R}^{n}\right)} & \leq C(1+j T) \exp (-3 \mu(j-1) T)\|\vec{f}\|_{\mathscr{H}^{k}(\Omega)} \\
& \leq C \exp (-2 \mu(j-1) T)\|\vec{f}\|_{\mathscr{H}^{k}(\Omega)},
\end{aligned}
$$

where $C$ is a constant depending on $k$ and $T$, but is independent of $j$. Here we have used the following elementary equality:

$$
\max _{y \in \boldsymbol{R}}(1+T+y) \exp (-\mu y)=\mu^{-1} \exp (\mu(1+T)-1) .
$$

Therefore we have

$$
\left\|\vec{f}_{+}\right\|_{\mathscr{H}^{k}\left(\boldsymbol{R}^{n}\right)} \leq \sum_{j=1}^{\infty} C(\exp (-2 \mu T))^{j-1}\|\vec{f}\|_{\mathscr{H}^{k}(\Omega)} \leq C\|\vec{f}\|_{\mathscr{H}^{k}(\Omega)}
$$


Step 2: Proof of (1.10) for fixed $k$. First we observe that the Huygens principle leads to

$$
\operatorname{supp}\left(U_{0}(t-j T) \vec{g}_{j}\right) \subset\left\{x \in \boldsymbol{R}^{n} ;|| x|-| j T-t|| \leq T+a_{*}\right\}
$$

for any natural number $j$ and $t \in \boldsymbol{R}$, because we have supp $\vec{g}_{j} \subset \overline{B_{T+a_{*}}}$.

For $\vec{h}=\left(h_{0}, h_{1}\right)$, we write

$$
|\vec{h}(x)|_{k}=\sum_{|\alpha| \leq k+1}\left|\partial_{x}^{\alpha} h_{0}(x)\right|+\sum_{|\alpha| \leq k}\left|\partial_{x}^{\alpha} h_{1}(x)\right|
$$

in what follows. Using the Sobolev imbedding theorem, from (3.5) and (3.7) with $t=0$, we get

$$
\left|\left(U_{0}(-j T) \vec{g}_{j}\right)(x)\right|_{k} \leq C \exp (-2 \mu|x|)\|\vec{f}\|_{\mathscr{H}^{k+[n / 2]+1}(\Omega)},
$$

where $C$ is a constant depending on $k, a$ and $T$, but is independent of $j$ and $x$. (1.10) follows from (3.9). Indeed, for each fixed $x \in \Omega$, the number of $j$ for which we have $x \in \operatorname{supp}\left(U_{0}(-j T) \vec{g}_{j}\right)$ is at most $\left[2\left(T+a_{*}\right) / T\right]+1$ because of (3.7). Therefore, remembering (3.4), we can sum up (3.9) to obtain

$$
\left|\vec{f}_{+}(x)\right|_{k} \leq C \exp (-2 \mu|x|)\|\vec{f}\|_{\mathscr{H}^{k+[n / 2]+1}(\Omega)}
$$

which shows (1.10).

Step 3: Proof of (1.9) for fixed $k$. For $t \geq T$, we are able to find a positive integer $J$ such that $t \in[J T,(J+1) T)$. By (3.1) with $j=1, \ldots, J$ we have

$$
U(t) \vec{f}=\left.\sum_{j=1}^{J}\left(U_{0}(t-j T) \vec{g}_{j}\right)\right|_{\Omega}+U(t-J T) \vec{f}_{J}
$$

By (3.4) we get

$$
\begin{aligned}
& \left\|e^{\mu<\cdot>}\left(U(t) \vec{f}-\left.\left(U_{0}(t) \vec{f}_{+}\right)\right|_{\Omega}\right)\right\|_{\mathscr{H}^{k}(\Omega)} \\
& \quad \leq \sum_{j=J+1}^{\infty}\left\|e^{\mu<\cdot\rangle} U_{0}(t-j T) \vec{g}_{j}\right\|_{\mathscr{H}^{k}(\Omega)}+\left\|e^{\mu<\cdot\rangle} U(t-J T) \vec{f}_{J}\right\|_{\mathscr{H}^{k}(\Omega)} .
\end{aligned}
$$

Note that (3.10) is also valid for $0 \leq t<T$, by regarding $J=0$ and $\vec{f}_{0}=\vec{f}$. So we assume $J \geq 0$ and $t \in[J T,(J+1) T)$ in the following.

First we evaluate the second term on the right-hand side of (3.10). Since $\operatorname{supp} \vec{f}_{J} \subset \overline{B_{3}}$ and $|t-J T| \leq T$ for $t \in[J T,(J+1) T)$, we have $|x| \leq T+3$ for $x \in \operatorname{supp}\left(U(t-J T) \vec{f}_{J}\right)$, and hence $\sum_{|\alpha| \leq k+1}\left|\partial_{x}^{\alpha} e^{\mu\langle x\rangle}\right| \leq C$ with some positive constant $C=C(k, a, T)$. Therefore, using (2.7) and (3.3), we get

$$
\left\|e^{\mu<\cdot\rangle} U(t-J T) \vec{f}_{J}\right\|_{\mathscr{H}^{k}(\Omega)} \leq C(1+T) \exp (-3 \mu J T)\|\vec{f}\|_{\mathscr{H}^{k}(\Omega)} .
$$


Next we estimate the first term on the right-hand side of (3.10). Let $j \geq J+1$. From (3.7) we have $|x| \leq T+a_{*}+j T-t$ for $x \in \operatorname{supp}\left(U_{0}(t-j T) \vec{g}_{j}\right)$, so that $\sum_{|\alpha| \leq k+1}\left|\partial_{x}^{\alpha} e^{\mu\langle x\rangle}\right| \leq C e^{\mu j T}$ with some positive constant $C=C(k, a, T)$. Hence, it follows from (2.6) and (3.2) that

$$
\begin{aligned}
\left\|e^{\mu<\cdot>} U_{0}(t-j T) \vec{g}_{j}\right\|_{\mathscr{H}^{k}(\Omega)} & \leq C e^{\mu j T}(1+|t-j T|)\left\|\vec{g}_{j}\right\|_{\mathscr{H}^{k}\left(\boldsymbol{R}^{n}\right)} \\
& \leq C(1+j T) e^{\mu j T-3 \mu(j-1) T}\|\vec{f}\|_{\mathscr{H}^{k}(\Omega)} \\
& \leq C e^{-\mu(j-1) T}\|\vec{f}\|_{\mathscr{H}^{k}(\Omega)},
\end{aligned}
$$

where $C$ is a constant independent of $j$ and $J$. Thus we get

$$
\sum_{j=J+1}^{\infty}\left\|e^{\mu<\cdot>} U_{0}(t-j T) \vec{g}_{j}\right\|_{\mathscr{H}^{k}(\Omega)} \leq C \exp (-\mu J T)\|\vec{f}\|_{\mathscr{H}^{k}(\Omega)},
$$

where $C$ is a constant independent of $J$. Finally by (3.11) and (3.12) we see that (1.9) holds for $t \in[J T,(J+1) T)$ with $J \geq 0$, and hence for all $t \geq 0$.

Step 4: Conclusion. For each fixed $k$, we have constructed $f_{+}$satisfying (1.9) and (1.10) for this fixed $k$. Observe that the construction itself depends on $k$ through the choice of $T$. However, by using Lemma 2.7, we can show that $\vec{f}_{+}$being constructed in the above is independent of $k$. In fact, let $\vec{f}_{+}^{(1)}$ and $\vec{f}_{+}^{(2)}$ denote $\vec{f}_{+}$constructed in the above with the choice of $k=k_{1}$ and $k=k_{2}$, respectively, where $k_{1}$ and $k_{2}$ are nonnegative integers. Then, from (1.9) we see that $(2.33)$ is valid for $\vec{f}_{+}=\vec{f}_{+}^{(1)}$ and $\vec{f}_{+}=\vec{f}_{+}^{(2)}$. Hence Lemma 2.7 implies $\vec{f}_{+}^{(1)}=\vec{f}_{+}^{(2)}$.

Now we find that the same $\vec{f}_{+}$enjoys the estimates (1.9) and (1.10) for all

$k$. This completes the proof of Theorem 1.1.

\section{The Friedlander radiation field for rapidly decreasing data}

Our aim in this section is to discuss the Friedlander radiation field for the Cauchy problem with rapidly decreasing data. The case of compactly supported data is well known (see Friedlander [2, 3, 4]; see also Hörmander [6] and John [8]). Indeed, for any $\vec{g} \in\left(C_{0}^{\infty}\left(\boldsymbol{R}^{n}\right)\right)^{2}$ and any multi-index $\alpha$, there exists a positive constant $C=C(\alpha, \vec{g})$ such that

$$
\begin{aligned}
& \left|Z^{\alpha}\left\{u_{0}(t, x)-r^{-(n-1) / 2} \widetilde{F}_{0}[\vec{g}](r-t, \omega)\right\}\right| \\
& \quad \leq C(1+t+r)^{-(n+1) / 2}(1+|r-t|)^{-(3-n) / 2},
\end{aligned}
$$

holds for $r \geq t / 2 \geq 1$ with $r=|x|$ and $\omega=r^{-1} x$, where $u_{0}(t, \cdot)$ is the first component of $U_{0}(t) \vec{g}$, and the radiation field $\mathscr{F}_{0}[\vec{g}](s, \eta)$ is given by $(1.11)$ (here 
$n$ can be either odd or even). The case of rapidly decreasing data was also treated in [6] through the conformal compactification of the Minkowski space, and (4.1) is valid also for this case. However the decay away from the light cone in (4.1) seems not to be sharp. For this reason, we would like to extend (4.1) in the two directions described below, restricting our attention to the odd space dimensional case.

The one is to give an argument to treat the case of rapidly decreasing data based on the explicit representation of $u_{0}$. This requires us to evaluate the spherical means over the whole sphere. In contrast, it is enough to estimate the integral over the portion of the sphere if the support of the data is compact.

The other is to extract a more detailed decay property away from the cone, in accordance with the stronger decay property of the scattering data $\vec{f}_{+}$ obtained in Theorem 1.1 than general functions in $\mathscr{S}\left(\boldsymbol{R}^{n}\right)$, where $\mathscr{S}\left(\boldsymbol{R}^{n}\right)$ is the Schwartz class, the set of rapidly decreasing functions. To this end, we introduce the following class of the data. Throughout this section, $\chi=\chi(s)$ is some given non-decreasing function of $s \geq 0$, satisfying $\chi(s) \geq 1$ for all $s \geq 0$. For $\varphi \in C^{\infty}\left(\boldsymbol{R}^{n}\right), m \geq 0$ and a nonnegative integer $k$, we define

$$
\|\varphi\|_{\chi, k, m}=\left(\sup _{x \in \boldsymbol{R}^{n}} \sum_{|\alpha| \leq k}\left(1+|x|^{2}\right)^{m} \chi^{2}(|x|)\left|\partial_{x}^{\alpha} \varphi(x)\right|^{2}\right)^{1 / 2},
$$

and let $\mathscr{S}_{\chi}\left(\boldsymbol{R}^{n}\right)$ be the set of all $\varphi \in C^{\infty}\left(\boldsymbol{R}^{n}\right)$ satisfying $\|\varphi\|_{\chi, m, k}<\infty$ for any nonnegative integers $m$ and $k$. Apparently we have $\mathscr{S}_{\chi}\left(\boldsymbol{R}^{n}\right) \subset \mathscr{S}\left(\boldsymbol{R}^{n}\right)$. Note that $\mathscr{S}_{\chi}\left(\boldsymbol{R}^{n}\right)=\mathscr{S}\left(\boldsymbol{R}^{n}\right)$ if $\chi$ is identically equal to 1 .

Our main result in this section is the following.

Proposition 4.1. Let $n$ be an odd integer with $n \geq 3$, and let $v \geq 0$. For any $\vec{g} \in\left(\mathscr{S}_{\chi}\left(\boldsymbol{R}^{n}\right)\right)^{2}$ and any multi-index $\alpha$, there exists a positive constant $C=$ $C(\alpha, v, \vec{g})$ such that we have

$$
\begin{aligned}
&\left|Z^{\alpha}\left\{u_{0}(t, x)-r^{-(n-1) / 2} \mathscr{F}_{0}[\vec{g}](r-t, \omega)\right\}\right| \\
& \leq C(1+t+r)^{-(n+1) / 2}(1+|r-t|)^{-v} \chi^{-1}(|r-t|), \\
&\left|Z^{\alpha}\left\{\partial_{t} u_{0}(t, x)-(-1) r^{-(n-1) / 2}\left(\partial_{s} \mathscr{F}_{0}[\vec{g}]\right)(r-t, \omega)\right\}\right| \\
& \quad+\sum_{j=1}^{n}\left|Z^{\alpha}\left\{\partial_{j} u_{0}(t, x)-\omega_{j} r^{-(n-1) / 2}\left(\partial_{s} \mathscr{F}_{0}[\vec{g}]\right)(r-t, \omega)\right\}\right| \\
& \leq C(1+t+r)^{-(n+1) / 2}(1+|r-t|)^{-v} \chi^{-1}(|r-t|)
\end{aligned}
$$

for $r \geq t / 2 \geq 1$ with $r=|x|$ and $\omega=\left(\omega_{1}, \ldots, \omega_{n}\right)=r^{-1} x$, where $u_{0}(t, \cdot)$ is the first component of $U_{0}(t) \vec{g}$. 
Before proceeding to prove Proposition 4.1, we give preliminaries about the Radon transform and spherical means in subsections 4.1 and 4.2, respectively, because the radiation field $\mathscr{F}_{0}[\vec{g}]$ and the solution $U_{0}(t) \vec{g}$ are closely related to the Radon transform and the spherical means, respectively.

\subsection{Basic properties of the Radon transform}

The Radon transform $\mathscr{R}[\varphi](s, \eta)$ for $\varphi \in \mathscr{S}\left(\boldsymbol{R}^{n}\right)$ is defined by

$$
\mathscr{R}[\varphi](s, \eta)=\int_{\Pi(s, \eta)} \varphi(y) d S_{y}, \quad(s, \eta) \in \boldsymbol{R} \times S^{n-1},
$$

where $\Pi(s, \eta)=\left\{y \in \boldsymbol{R}^{n} ; y \cdot \eta=s\right\}$, and $d S_{y}$ denotes the area element on $\Pi(s, \eta)$. For $\eta \in S^{n-1}$ and a smooth function $\varphi=\varphi(y)$ on $\boldsymbol{R}^{n}, D_{\eta} \varphi$ denotes the directional derivative of $\varphi$ in the direction $\eta$; in other words, we define $\left(D_{\eta} \varphi\right)(y)=$ $\eta \cdot \nabla_{y} \varphi(y)$. We write

$$
o_{i j}=\eta_{i} \partial_{\eta_{j}}-\eta_{j} \partial_{\eta_{i}}, \quad 1 \leq i, j \leq n .
$$

We put $o=\left(o_{1}, \ldots, o_{n(n-1) / 2}\right)=\left(o_{i j}\right)_{1 \leq i<j \leq n}$, where $o_{i j}$ 's are regarded to be arranged in dictionary order. We write $o^{\alpha}=o_{1}^{\alpha_{1}} \ldots o_{d}^{\alpha_{d}}$ with a multi-index $\alpha=$ $\left(\alpha_{1}, \ldots, \alpha_{d}\right)$, where $d=n(n-1) / 2 . \quad O^{\alpha}$ is similarly defined using $O_{i j}$ instead of $o_{i j}$, where $\left(O_{i j} \varphi\right)(y)=y_{i}\left(\partial_{j} \varphi\right)(y)-y_{j}\left(\partial_{i} \varphi\right)(y)$ as before.

It is easy to check

$$
\begin{aligned}
& \partial_{s} \mathscr{R}[\varphi](s, \eta)=\mathscr{R}\left[D_{\eta} \varphi\right](s, \eta)\left(=\int_{\Pi(s, \eta)}\left(D_{\eta} \varphi\right)(y) d S_{y}\right), \\
& o_{i j} \mathscr{R}[\varphi](s, \eta)=\mathscr{R}\left[O_{i j} \varphi\right](s, \eta), \quad 1 \leq i<j \leq n
\end{aligned}
$$

for $\varphi \in \mathscr{S}\left(\boldsymbol{R}^{n}\right)$. Because integrals over $\Pi(s, \eta)$ of directional derivatives of $\varphi$ in directions proportional to $\Pi(s, \eta)$ vanish, we get

$$
\mathscr{R}\left[\partial_{i} \varphi\right](s, \eta)=\mathscr{R}\left[\eta_{i} D_{\eta} \varphi\right](s, \eta)=\eta_{i} \partial_{s} \mathscr{R}[\varphi](s, \eta)
$$

for $1 \leq i \leq n$. Moreover, we have the following.

Lemma 4.2. Let $\varphi \in \mathscr{S}_{\chi}\left(\boldsymbol{R}^{n}\right), v \geq 0, k$ be a nonnegative integer, and $\alpha, \beta$ be multi-indices. Then we have

$$
\left|\partial_{s}^{k} o^{\alpha} \mathscr{R}[\varphi](s, \eta)\right| \leq C_{k, \alpha}\|\varphi\|_{\chi, k+|\alpha|, v+n+|\alpha|}\left(1+s^{2}\right)^{-v / 2} \chi^{-1}(|s|)
$$


for $(s, \eta) \in \boldsymbol{R} \times S^{n-1}$, and

$$
\begin{gathered}
\left|\partial_{x}^{\alpha} O^{\beta}\left\{\left(\partial_{s}^{k} \mathscr{R}[\varphi]\right)\left(|x|-t, \frac{x}{|x|}\right)\right\}-\left(\partial_{s}^{k} \mathscr{R}\left[\partial_{x}^{\alpha} O^{\beta} \varphi\right]\right)\left(|x|-t, \frac{x}{|x|}\right)\right| \\
\leq C \frac{\|\varphi\|_{\chi, k+|\alpha|+|\beta|, v+n+|\alpha|+|\beta|}}{(1+t+|x|)(1+|| x|-t|)^{v} \chi(|| x|-t|)}
\end{gathered}
$$

for $|x| \geq t / 2 \geq 1$. Here $C=C(k, v, \alpha, \beta)$ is a positive constant.

Proof. First we prove (4.7). Writing $\rho=|y-(y \cdot \eta) \eta|$, we have $|y|^{2}=$ $s^{2}+\rho^{2}$ for $y \in \Pi(s, \eta)$. Hence we get

$$
\left|\left(D_{\eta}^{k} O^{\alpha} \varphi\right)(y)\right| \leq C_{k, \alpha}\left(1+s^{2}+\rho^{2}\right)^{-v / 2}(1+\rho)^{-n} \chi^{-1}(|s|)\|\varphi\|_{\chi, k+|\alpha|, v+n+|\alpha|}
$$

for $y \in \Pi(s, \eta)$. Using (4.4) and (4.5), we obtain (4.7).

Next we prove (4.8). Since $O^{\beta}\left\{\psi\left(|x|^{-1} x\right)\right\}=\left(o^{\beta} \psi\right)\left(|x|^{-1} x\right)$ for any $\psi \in$ $C^{\infty}\left(S^{n-1}\right)$, it suffices to show (4.8) for $\beta=0$, thanks to (4.5). Besides, it is clear that (4.8) holds for $\alpha=\beta=0$. By (4.6) we have

$$
\begin{aligned}
\partial_{i}\left\{\left(\partial_{s}^{k} \mathscr{R}[\varphi]\right)\left(|x|-t,|x|^{-1} x\right)\right\} \\
\quad=\left.\left(\eta_{i} \partial_{s}^{k+1} \mathscr{R}[\varphi](s, \eta)-|x|^{-1} \sum_{j=1}^{n} \eta_{j} o_{i j} \partial_{s}^{k} \mathscr{R}[\varphi](s, \eta)\right)\right|_{(s, \eta)=\left(|x|-t,|x|^{-1} x\right)} \\
\quad=\left(\partial_{s}^{k} \mathscr{R}\left[\partial_{i} \varphi\right]\right)\left(|x|-t,|x|^{-1} x\right)-\sum_{j=1}^{n} \frac{x_{j}}{|x|^{2}}\left(o_{i j} \partial_{s}^{k} \mathscr{R}[\varphi]\right)\left(|x|-t,|x|^{-1} x\right)
\end{aligned}
$$

for $1 \leq i \leq n$ and any nonnegative integer $k$. Therefore, (4.7) implies

$$
\begin{gathered}
\left|\partial_{i}\left\{\left(\partial_{s}^{k} \mathscr{R}[\varphi]\right)\left(|x|-t,|x|^{-1} x\right)\right\}-\left(\partial_{s}^{k} \mathscr{R}\left[\partial_{i} \varphi\right]\right)\left(|x|-t,|x|^{-1} x\right)\right| \\
\leq C \frac{\|\varphi\|_{\chi, k+1, v+n+1}}{(1+t+|x|)(1+|| x|-t|)^{v} \chi(|| x|-t|)}
\end{gathered}
$$

for $|x| \geq t / 2 \geq 1,1 \leq i \leq n$, and $v \geq 0$, where $C$ is a positive constant depending on $k$ and $v$. Hence (4.8) holds for $|\alpha|=1$. We can show (4.8) for general $\alpha$ in a similar manner. This completes the proof.

\subsection{Spherical means}

Let $n(\geq 3)$ be an odd integer. We define

$$
Q[\varphi](t, x)=\frac{1}{A_{n}} \int_{\theta \in S^{n-1}} \varphi(x+t \theta) d S_{\theta}^{\prime}
$$


for $\varphi \in \mathscr{S}\left(\boldsymbol{R}^{n}\right)$ and $(t, x) \in(0, \infty) \times \boldsymbol{R}^{n}$. Here $A_{n}$ is the total measure of $S^{n-1}$, i.e., $A_{n}=2 \pi^{n / 2} / \Gamma(n / 2)$ with the Gamma function $\Gamma$, and $d S_{\theta}^{\prime}$ is the area element on $S^{n-1}$. $Q[\varphi]$ is the spherical mean of $\varphi$. The aim of this subsection is to prove the following.

Lemma 4.3. Let $\ell$ be a nonnegative integer, and $v \geq 0$. Then there exist a positive constant $C$ and a large integer $N$ such that we have

$$
\begin{aligned}
& \left|A_{n} t^{n-1} \partial_{t}^{\ell} Q[\varphi](t, x)-\left(\left(-\partial_{s}\right)^{\ell} \mathscr{R}[\varphi]\right)(r-t, \omega)\right| \\
& \quad \leq C\|\varphi\|_{\chi, \ell+1, N}(1+t+r)^{-1}(1+|r-t|)^{-v} \chi^{-1}(|r-t|)
\end{aligned}
$$

for $(t, x) \in(0, \infty) \times \boldsymbol{R}^{n}$ satisfying $r \geq t / 2 \geq 1$ with $r=|x|$ and $\omega=x /|x|$, provided that $\varphi \in \mathscr{S}_{\chi}\left(\boldsymbol{R}^{n}\right)$.

To prove Lemma 4.3, we first make some reduction: For $\varphi=\varphi(y)$, let $D_{\theta} \varphi$ be the directional derivative of $\varphi$ in the direction $\theta$ as before, and let $c_{\ell, \alpha}(\theta)$ be the coefficient for $\partial_{y}^{\alpha}$ in the expansion of $D_{\theta}^{\ell}$ :

$$
D_{\theta}^{\ell}=\left(\theta \cdot \partial_{y}\right)^{\ell}=\sum_{|\alpha|=\ell} c_{\ell, \alpha}(\theta) \partial_{y}^{\alpha},
$$

so that $c_{\ell, \alpha}$ is a homogeneous polynomial of degree $\ell$. Then it follows from (4.10) that

$$
\begin{aligned}
\partial_{t}^{\ell} Q[\varphi](t, x) & =\frac{1}{A_{n}} \int_{S^{n-1}}\left(D_{\theta}^{\ell} \varphi\right)(x+t \theta) d S_{\theta}^{\prime} \\
& =\frac{1}{A_{n}} \sum_{|\alpha|=\ell} \int_{S^{n-1}} c_{\ell, \alpha}(\theta)\left(\partial_{y}^{\alpha} \varphi\right)(x+t \theta) d S_{\theta}^{\prime} \\
& =\frac{1}{A_{n} t^{n-1}} \sum_{|\alpha|=\ell} \int_{S(t, x)} c_{\ell, \alpha}\left(t^{-1}(y-x)\right) \partial_{y}^{\alpha} \varphi(y) d S_{y}^{*}
\end{aligned}
$$

where $S(t, x)=\left\{y \in \boldsymbol{R}^{n} ;|y-x|=t\right\}$ and $d S_{y}^{*}$ stands for the area element on $S(t, x)$. On the other hand, (4.4) implies that

$$
\left(-\partial_{s}\right)^{\ell} \mathscr{R}[\varphi](r-t, \omega)=\sum_{|\alpha|=\ell} c_{\ell, \alpha}(-\omega) \mathscr{R}\left[\partial_{y}^{\alpha} \varphi\right](r-t, \omega) .
$$

Thus the proof of (4.11) is reduced to proving

$$
\begin{gathered}
\left|\int_{S(t, x)} c_{\ell, \alpha}\left(t^{-1}(y-x)\right) \partial_{y}^{\alpha} \varphi(y) d S_{y}^{*}-c_{\ell, \alpha}(-\omega) \mathscr{R}\left[\partial_{y}^{\alpha} \varphi\right](r-t, \omega)\right| \\
\leq C\|\varphi\|_{\chi,|\alpha|+1, N}(1+t+r)^{-1}(1+|r-t|)^{-v} \chi^{-1}(|r-t|)
\end{gathered}
$$


for $r \geq t / 2 \geq 1$. In order to proceed further, we decompose $S(t, x)$ as follows: Let $\varepsilon$ be a small and positive constant. For $r>0, t>0$ and $\omega \in S^{n-1}$, we set

$$
\begin{aligned}
& \Lambda_{\varepsilon}^{1}(t, r, \omega)=\left\{y \in S(t, r \omega) ;|y|>(t+r)^{\varepsilon}\right\}, \\
& \Lambda_{\varepsilon}^{2}(t, r, \omega)=\left\{y \in S(t, r \omega) ;|y| \leq(t+r)^{\varepsilon}\right\} .
\end{aligned}
$$

We are going to prove that the integral over $\Lambda_{\varepsilon}^{1}$ can be regarded as the remainder term (Lemma 4.4), while the integral over $\Lambda_{\varepsilon}^{2}$ can be approximated by the Radon transform (Lemma 4.5). Using these estimates we will show (4.14) to finish the proof of Lemma 4.3.

Lemma 4.4. Let $c$ be a bounded function on $S^{n-1}$, and $\varphi \in \mathscr{S}_{\chi}\left(\boldsymbol{R}^{n}\right)$. Let $\varepsilon>0$ and $\kappa>0$. Suppose that $N_{1}$ is a positive integer satisfying $N_{1} \varepsilon \geq$ $\kappa+n-1$. Then there exists a positive constant $C=C\left(\varepsilon, \kappa, N_{1}\right)$ such that we have

$\left|\int_{\Lambda_{\varepsilon}^{1}(t, r, \omega)} c\left(t^{-1}(y-r \omega)\right) \varphi(y) d S_{y}^{*}\right| \leq C(1+t+r)^{-\kappa} \chi^{-1}(|r-t|)\|c\|_{L^{\infty}\left(S^{n-1}\right)}\|\varphi\|_{\chi, 0, N_{1}}$ for any $(t, r, \omega) \in[0, \infty) \times[0, \infty) \times S^{n-1}$.

Proof. Since we have $|y| \geq \max \left\{|r-t|,(t+r)^{\varepsilon}\right\}$ for $y \in \Lambda_{\varepsilon}^{1}(t, r, \omega)$, we get

$$
\begin{aligned}
|\varphi(y)| & \leq\left(1+|t+r|^{2 \varepsilon}\right)^{-N_{1} / 2} \chi^{-1}(|r-t|)\|\varphi\|_{\chi, 0, N_{1}} \\
& \leq C(1+t+r)^{-\kappa-n+1} \chi^{-1}(|r-t|)\|\varphi\|_{\chi, 0, N_{1}}
\end{aligned}
$$

for any $y \in \Lambda_{\varepsilon}^{1}(t, r, \omega)$ with some positive constant $C$. Observing that the total measure of $S(t, r \omega)$ is bounded by $A_{n} t^{n-1}$, we obtain the desired result from (4.15).

Lemma 4.5. Let $c \in C^{1}\left(\overline{B_{1}}\right)$, and $\varphi \in \mathscr{S}_{\chi}\left(\boldsymbol{R}^{n}\right)$. Let $0<\varepsilon \leq 1 / 4$, and $v \geq 0$. Suppose that $N_{2}$ is a positive integer satisfying $N_{2} \geq n+2+v+(1 / \varepsilon)$. Then there exists a positive constant $C=C\left(\varepsilon, v, N_{2}\right)$ such that we have

$$
\begin{aligned}
& \left|\int_{\Lambda_{\varepsilon}^{2}(t, r, \omega)} c\left(t^{-1}(y-r \omega)\right) \varphi(y) d S_{y}^{*}-c(-\omega) \mathscr{R}[\varphi](r-t, \omega)\right| \\
& \quad \leq C(1+t+r)^{-1}(1+|r-t|)^{-v} \chi^{-1}(|r-t|)\|c\|_{C^{1}\left(\overline{B_{1}}\right)}\|\varphi\|_{\chi, 1, N_{2}}
\end{aligned}
$$

for any $(t, r, \omega) \in[0, \infty) \times[0, \infty) \times S^{n-1}$ with $r \geq t / 2 \geq 1$ and $|r-t| \leq(t+r)^{\varepsilon}$, where

$$
\|\psi\|_{C^{1}\left(\overline{B_{1}}\right)}=\sup _{y \in \overline{B_{1}}}\left(|\psi(y)|^{2}+\left|\nabla_{y} \psi(y)\right|^{2}\right)^{1 / 2}
$$

for $\psi \in C^{1}\left(\overline{B_{1}}\right)$. 
Proof. Since the right-hand side of (4.16) is invariant under the orthogonal transforms, we may assume $\omega=e_{n}$ without loss of generality, where $e_{n}=$ $(0, \ldots, 0,1)$. In the following, we suppose $r \geq t / 2 \geq 1,|r-t| \leq(t+r)^{\varepsilon}$ and $0<\varepsilon \leq 1 / 4$.

Since $t+r \geq 3$, we get $(t+r)^{\varepsilon-1} \leq 3^{-3 / 4}<1 / 2$, which implies

$$
(t+r)^{\varepsilon}<\frac{t+r}{2}
$$

From (4.17) we also get $r \leq 3 t$, because otherwise (namely $r>3 t$ ) we get $r-t>(t+r) / 2>(t+r)^{\varepsilon}$, which contradicts the assumption. Summing up, we obtain

$$
1 \leq \frac{t}{2} \leq r \leq 3 t
$$

and we find that $t, r$ and $1+t+r$ are equivalent to each other.

First we prove that

$$
\begin{aligned}
& \left|\int_{\Lambda_{\varepsilon}^{2}\left(t, r, e_{n}\right)} c\left(t^{-1}\left(y-r e_{n}\right)\right) \varphi(y) d S_{y}^{*}-c\left(-e_{n}\right) \int_{\Lambda_{\varepsilon}^{2}\left(t, r, e_{n}\right)} \varphi(y) d S_{y}^{*}\right| \\
& \quad \leq 2^{(n-2) / 2} A_{n-1}\|c\|_{C^{1}\left(\overline{B_{1}}\right)}\|\varphi\|_{\chi, 1, N_{2}} t^{-1}\left(1+|r-t|^{2}\right)^{-v / 2} \chi^{-1}(|r-t|) .
\end{aligned}
$$

We put

$$
\lambda_{0}(t, r)=\frac{(t+r)^{2 \varepsilon}-(r-t)^{2}}{2 r t} .
$$

Note that $0 \leq \lambda_{0}(t, r) \leq 1$. Writing $t \lambda=y_{n}-(r-t)$, we find that $\Lambda_{\varepsilon}^{2}\left(t, r, e_{n}\right)$ is equal to

$$
\left\{y=(t \sqrt{\lambda(2-\lambda)} \zeta, r-t+t \lambda) ; \zeta \in S^{n-2}, 0 \leq \lambda \leq \lambda_{0}(t, r)\right\} .
$$

For the coordinate system $(\zeta, \lambda)$ in the above, we have

$$
d S_{y}^{*}=t^{n-1} \lambda^{(n-3) / 2}(2-\lambda)^{(n-3) / 2} d \lambda d S_{\zeta}^{\prime \prime}
$$

where $d S_{\zeta}^{\prime \prime}$ denotes the area element on $S^{n-2}$. We also note that

$$
|y|^{2}=(r-t)^{2}+2 r t \lambda
$$

We put $\eta(y, r)=\left|y-r e_{n}\right|^{-1}\left(y-r e_{n}\right) \in S^{n-1}$. Then we get

$$
\left|\eta(y, r)-\left(-e_{n}\right)\right|=\sqrt{2+2 \eta_{n}(y, r)}=\sqrt{2 \lambda}
$$


for any $y \in \Lambda_{\varepsilon}^{2}\left(t, r, e_{n}\right)$. Hence, by the mean value theorem, we get

$$
\left|\int_{{\Lambda_{\varepsilon}}^{2}\left(t, r, e_{n}\right)}\left\{c(\eta(y, r))-c\left(-e_{n}\right)\right\} \varphi(y) d S_{y}^{*}\right| \leq \sqrt{2}\|c\|_{C^{1}\left(\overline{B_{1}}\right)} J(t, r),
$$

where we put

$$
J(t, r)=\int_{\Lambda_{\varepsilon}^{2}\left(t, r, e_{n}\right)} \lambda^{1 / 2}|\varphi(y)| d S_{y}^{*}
$$

with $t \lambda=y_{n}-(r-t)$. Note that we have $\eta(y, r)=t^{-1}\left(y-r e_{n}\right)$ for $y \in$ $\Lambda_{\varepsilon}^{2}\left(t, r, e_{n}\right)$. Recalling (4.20) and (4.21), we obtain

$$
\begin{aligned}
\chi(\mid r- & t \mid) J(t, r) \\
\leq & A_{n-1} t^{n-1}\|\varphi\|_{\chi, 0, N_{2}} \int_{0}^{\lambda_{0}(t, r)} \frac{\lambda^{(n-2) / 2}(2-\lambda)^{(n-3) / 2}}{\left(1+(r-t)^{2}+2 r t \lambda\right)^{N_{2} / 2}} d \lambda \\
\leq & 2^{-1 / 2} A_{n-1} r\left(\frac{t}{r}\right)^{n / 2}\|\varphi\|_{\chi, 0, N_{2}} \\
& \times \int_{0}^{\lambda_{0}(t, r)} \frac{(2 r t \lambda)^{(n-2) / 2}}{\left(1+|r-t|^{2}\right)^{v / 2}(1+2 r t \lambda)^{\left(N_{2}-v\right) / 2}} d \lambda \\
\leq & 2^{(n-1) / 2} A_{n-1} r\left(1+|r-t|^{2}\right)^{-v / 2}\|\varphi\|_{\chi, 0, N_{2}} \int_{0}^{\infty} \frac{1}{(1+2 r t \lambda)^{2}} d \lambda \\
\leq & 2^{(n-3) / 2} A_{n-1} t^{-1}\left(1+|r-t|^{2}\right)^{-v / 2}\|\varphi\|_{\chi, 0, N_{2}} \cdot
\end{aligned}
$$

This estimate yields (4.19) immediately.

By (4.19), we find that, in order to show (4.16), it suffices to prove

$$
\begin{aligned}
& \left|\int_{\Lambda_{\varepsilon}^{2}\left(t, r, e_{n}\right)} \varphi(y) d S_{y}^{*}-\mathscr{R}[\varphi]\left(r-t, e_{n}\right)\right| \\
& \quad \leq C t^{-1}(1+|r-t|)^{-v} \chi^{-1}(|r-t|)\|\varphi\|_{\chi, 1, N_{2}}
\end{aligned}
$$

with some positive constant $C$. We observe that

$$
\left|\int_{\Lambda_{\varepsilon}^{2}\left(t, r, e_{n}\right)} \lambda \varphi(y) d S_{y}^{*}\right| \leq C(r t)^{-1}(1+|r-t|)^{-v} \chi^{-1}(|r-t|)\|\varphi\|_{\chi, 0, N_{2}},
$$

which can be shown similarly to (4.22). Therefore we obtain (4.23), if we can show

$$
\left|I_{1}(r, t)-\mathscr{R}[\varphi]\left(r-t, e_{n}\right)\right| \leq C t^{-1}(1+|r-t|)^{-v} \chi^{-1}(|r-t|)\|\varphi\|_{\chi, 1, N_{2}}
$$


where we put

$$
I_{1}(r, t)=\int_{\Lambda_{\varepsilon}^{2}\left(t, r, e_{n}\right)}(1-\lambda) \varphi(y) d S_{y}^{*} .
$$

Now we are going to prove (4.24). Introducing a new coordinate $\rho=$ $t \sqrt{\lambda(2-\lambda)}$, we get

$$
I_{1}(t, r)=\int_{S^{n-2}}\left(\int_{0}^{\rho_{0}(t, r)} \varphi\left(\rho \zeta, r-\sqrt{t^{2}-\rho^{2}}\right) \rho^{n-2} d \rho\right) d S_{\zeta}^{\prime \prime},
$$

where $\rho_{0}(t, r)=t \sqrt{\lambda_{0}(t, r)\left(2-\lambda_{0}(t, r)\right)}$. We also have

$$
\mathscr{R}[\varphi]\left(r-t, e_{n}\right)=I_{2}(t, r)+I_{3}(t, r),
$$

where we put

$$
\begin{aligned}
& I_{2}(t, r)=\int_{S^{n-2}}\left(\int_{0}^{\rho_{0}(t, r)} \varphi(\rho \zeta, r-t) \rho^{n-2} d \rho\right) d S_{\zeta}^{\prime \prime}, \\
& I_{3}(t, r)=\int_{S^{n-2}}\left(\int_{\rho_{0}(t, r)}^{\infty} \varphi(\rho \zeta, r-t) \rho^{n-2} d \rho\right) d S_{\zeta}^{\prime \prime} .
\end{aligned}
$$

Since $t-\sqrt{t^{2}-\rho^{2}}=\rho^{2}\left(t+\sqrt{t^{2}-\rho^{2}}\right)^{-1}$, we get

$$
\begin{aligned}
& \left|\varphi\left(\rho \zeta, r-\sqrt{t^{2}-\rho^{2}}\right)-\varphi(\rho \zeta, r-t)\right| \\
& \quad \leq\left(t-\sqrt{t^{2}-\rho^{2}}\right) \int_{0}^{1}\left|\left(\partial_{n} \varphi\right)\left(\rho \zeta, r-t+\tau\left(t-\sqrt{t^{2}-\rho^{2}}\right)\right)\right| d \tau \\
& \quad \leq \frac{\rho^{2}\|\varphi\|_{\chi, 1, N_{2}}}{t\left(1+|r-t|^{2}\right)^{v / 2}\left(1+\rho^{2}\right)^{(n+2) / 2} \chi(|r-t|)}
\end{aligned}
$$

for $0 \leq \rho \leq \rho_{0}(t, r)$, which yields

$$
\begin{aligned}
\left|I_{1}(t, r)-I_{2}(t, r)\right| & \leq \frac{A_{n-1}\|\varphi\|_{\chi, 1, N_{2}}}{t\left(1+|r-t|^{2}\right)^{v / 2} \chi(|r-t|)} \int_{0}^{\infty} \frac{\rho}{\left(1+\rho^{2}\right)^{3 / 2}} d \rho \\
& \leq C A_{n-1}\|\varphi\|_{\chi, 1, N_{2}} t^{-1}(1+|r-t|)^{-v} \chi^{-1}(|r-t|),
\end{aligned}
$$

where $C$ is a positive constant depending only on $v$.

Next we evaluate $I_{3}(t, r)$. Notice that

$$
\left(\rho_{0}(t, r)\right)^{2}+(r-t)^{2} \geq \frac{3}{32}(t+r)^{2 \varepsilon} .
$$


In fact, it is trivial when $(t+r)^{2 \varepsilon} / 2 \leq(r-t)^{2}\left(\leq(t+r)^{2 \varepsilon}\right)$. On the other hand, when $(r-t)^{2} \leq(t+r)^{2 \varepsilon} / 2$, by (4.17) we get

$$
\left(\rho_{0}(t, r)\right)^{2}=\frac{(t+r)^{2}-(t+r)^{2 \varepsilon}}{4 r^{2}}\left\{(t+r)^{2 \varepsilon}-(r-t)^{2}\right\} \geq \frac{3}{32}(t+r)^{2 \varepsilon},
$$

which shows (4.27). We thus find

$$
\begin{aligned}
\left|I_{3}(t, r)\right| & \leq \frac{A_{n-1}\|\varphi\|_{\chi, 0, N_{2}}}{\chi(|r-t|)} \int_{\rho_{0}(t, r)}^{\infty} \frac{\rho}{\left(1+\rho^{2}+(r-t)^{2}\right)^{(v+5+1 / \varepsilon) / 2}} d \rho \\
& \leq C\|\varphi\|_{\chi, 0, N_{2}}(t+r)^{-1}(1+|r-t|)^{-v} \chi^{-1}(|r-t|),
\end{aligned}
$$

where $C$ is a constant depending only on $v, n$ and $\varepsilon$.

Finally (4.24) follows from (4.25), (4.26) and (4.28). This completes the proof.

Now we are in a position to prove (4.14). When $|r-t| \leq(t+r)^{\varepsilon},(4.14)$ is an immediate consequence of Lemmas 4.4 and 4.5. On the other hand, when $|r-t|>(t+r)^{\varepsilon}$, we have $S(t, r \omega)=\Lambda_{\varepsilon}^{1}(t, r, \omega)$. Therefore, using Lemma 4.4 and (4.7) to estimate

$$
\int_{S(t, x)} c_{\ell, \alpha}\left(t^{-1}(y-x)\right) \partial_{y}^{\alpha} \varphi(y) d S_{y}^{*} \quad \text { and } \quad c_{\ell, \alpha}(-\omega) \mathscr{R}\left[\partial_{y}^{\alpha} \varphi\right](r-t, \omega),
$$

respectively, we obtain (4.14). This completes the proof of (4.14), and thus Lemma 4.3 is proved.

\subsection{Proof of Proposition 4.1}

Suppose that all the assumptions of the proposition are fulfilled.

First we recall the explicit representation of $u_{0}(t, \cdot)$, the first component of $U_{0}(t) \vec{g}$. We define

$$
E[\varphi](t, x):=\frac{\sqrt{\pi}}{2 \Gamma(n / 2)}\left(\frac{1}{2 t} \frac{\partial}{\partial t}\right)^{(n-3) / 2}\left(t^{n-2} Q[\varphi](t, x)\right),
$$

where $\Gamma(s)$ is the Gamma function and $Q[\varphi]$ is defined by (4.10). Then it is known that we have

$$
u_{0}(t, x)=\partial_{t} E\left[g_{0}\right](t, x)+E\left[g_{1}\right](t, x)
$$

for $\vec{g}=\left(g_{0}, g_{1}\right) \in\left(\mathscr{S}\left(\boldsymbol{R}^{n}\right)\right)^{2}$ (see, e.g., Courant and Hilbert [1, Chapter VI, Section 12]). Therefore we get

$$
\partial_{t}^{k} \partial_{x}^{\alpha} O^{\beta} u_{0}(t, x)=\partial_{t}^{k+1} E\left[\partial_{x}^{\alpha} O^{\beta} g_{0}\right](t, x)+\partial_{t}^{k} E\left[\partial_{x}^{\alpha} O^{\beta} g_{1}\right](t, x)
$$


for any nonnegative integer $k$ and any multi-indices $\alpha, \beta$ (note that $\varphi \in \mathscr{S}_{\chi}\left(\boldsymbol{R}^{n}\right)$ implies $\partial_{x}^{\alpha} O^{\beta} \varphi \in \mathscr{S}_{\chi}\left(\boldsymbol{R}^{n}\right)$ for any multi-indices $\alpha$ and $\left.\beta\right)$. Thus we find that our task is to evaluate $\partial_{t}^{k} E[\varphi](t, x)$ for $\varphi \in \mathscr{S}_{\chi}\left(\boldsymbol{R}^{n}\right)$ and a nonnegative integer $k$.

It is easy to see that

$$
\partial_{t}^{k} E[\varphi](t, x)=\frac{\sqrt{\pi}}{2^{(n-1) / 2} \Gamma(n / 2)} \sum_{\ell=0}^{(n-3) / 2+k} a_{k, \ell} t^{1-k+\ell} \partial_{t}^{\ell} Q[\varphi](t, x)
$$

for $k \geq 0$, where $a_{k, \ell}$ 's are suitable constants with $a_{k, \ell}=1$ for $\ell=(n-3) /$ $2+k$. Let $\varphi \in \mathscr{S}_{\chi}\left(\boldsymbol{R}^{n}\right), v \geq 0, r(=|x|) \geq t / 2 \geq 1$, and $\omega=r^{-1} x$ in the following. Then we find from (4.11) that there exist a large integer $N$ and a positive constant $C=C(k)$ such that

$$
\begin{aligned}
& \left|\partial_{t}^{k} E[\varphi](t, x)-\frac{1}{2(2 \pi r)^{(n-1) / 2}}\left(\left(-\partial_{s}\right)^{(n-3) / 2+k} \mathscr{R}[\varphi]\right)(r-t, \omega)\right| \\
& \leq C\|\varphi\|_{\chi,(n-1) / 2+k, N}(1+t+r)^{-(n+1) / 2}(1+|r-t|)^{-v} \chi^{-1}(|r-t|)
\end{aligned}
$$

holds. In fact, when $2 t \geq r \geq t / 2 \geq 1$, since we have

$$
\left|t^{-(n-1) / 2}-r^{-(n-1) / 2}\right| \leq C(1+t+r)^{-(n+1) / 2}(1+|r-t|),
$$

from (4.7) we see that it suffices to prove (4.33) with $(2 \pi r)^{(n-1) / 2}$ in its left-hand side being replaced by $(2 \pi t)^{(n-1) / 2}$. This replaced estimate can be easily proved by (4.32), (4.11) and (4.7), because $t$ is equivalent to $1+t+r$ in this case. On the other hand, when $r>2 t(\geq 4)$, from (4.7) we get

$$
\left|\left(\left(-\partial_{s}\right)^{\ell} \mathscr{R}[\varphi]\right)(r-t, \omega)\right| \leq C\|\varphi\|_{\chi, \ell, \mu+n}(1+t+r)^{-\mu} \chi^{-1}(|r-t|)
$$

for any $\mu \geq 0$ (note that $1+|r-t|$ is equivalent to $1+t+r$ in this case). Hence, using (4.32) and (4.11), we find that both $\left|\partial_{t}^{k} E[\varphi](t, x)\right|$ and $\left.\mid\left(-\partial_{s}\right)^{(n-3) / 2+k} \mathscr{R}[\varphi]\right)(r-t, \omega) \mid$ are bounded from above by the right-hand side of (4.33), provided that $N$ is sufficiently large.

Now, using (1.11), (4.6), (4.7), (4.8), (4.31), and (4.33), we obtain Proposition 4.1. This completes the proof.

\section{Proof of Theorem 1.2}

To begin with, we note that, for any $A \in \boldsymbol{R}$ and any nonnegative integer $k$, there exists a positive constant $C$ such that we have

$$
C^{-1} e^{A\langle x\rangle} \sum_{|\alpha| \leq k}\left|\partial_{x}^{\alpha} \psi(x)\right| \leq \sum_{|\alpha| \leq k}\left|\partial_{x}^{\alpha}\left(e^{A\langle x\rangle} \psi(x)\right)\right| \leq C e^{A\langle x\rangle} \sum_{|\alpha| \leq k}\left|\partial_{x}^{\alpha} \psi(x)\right|
$$


for any $x \in \boldsymbol{R}^{n}$ and any $\psi \in C^{\infty}\left(\boldsymbol{R}^{n}\right)$. In fact, the latter half is almost apparent, and the first half is nothing but the latter half with $A$ and $\psi(x)$ being replaced by $-A$ and $e^{A\langle x\rangle} \psi(x)$, respectively.

Let the assumptions in Theorem 1.2 be fulfilled. Then, by Theorem 1.1, there exists $f_{+} \in \mathscr{H}^{\infty}\left(\boldsymbol{R}^{n}\right)$ satisfying (1.9) and (1.10). We write $u(t, \cdot)$ and $u_{+}(t, \cdot)$ for the first components of $U(t) \vec{f}$ and $\left.\left(U_{0}(t) \vec{f}_{+}\right)\right|_{\Omega}$, respectively.

First we claim that we have

$$
\begin{gathered}
\left\|\exp (3 \mu\langle\cdot\rangle / 4)\left(Z^{\alpha} u(t, \cdot)-Z^{\alpha} u_{+}(t, \cdot)\right)\right\|_{H^{[n / 2]+1}(\Omega)} \\
\leq C \exp (-\mu t)\|\vec{f}\|_{\mathscr{H}^{[n / 2]+|\alpha|}(\Omega)}, \quad t \geq 0
\end{gathered}
$$

for any multi-index $\alpha$. Let $Z^{\alpha}=\partial_{t}^{j} \partial_{x}^{\beta} O^{\gamma}$ with a nonnegative integer $j$, and multi-indices $\beta, \gamma$, and let $\alpha^{\prime}$ be a multi-index satisfying $\left|\alpha^{\prime}\right| \leq[n / 2]+1$. If $j$ is even, then we get

$$
\begin{aligned}
\left|\partial_{x}^{\alpha^{\prime}} Z^{\alpha}\left(u-u_{+}\right)(t, x)\right| & =\left|\Delta^{j / 2} \partial_{x}^{\alpha^{\prime}+\beta} O^{\gamma}\left(u-u_{+}\right)(t, x)\right| \\
& \leq C(1+|x|)^{|\gamma|} \sum_{\left|\beta^{\prime}\right| \leq|\alpha|+\left|\alpha^{\prime}\right|}\left|\partial_{x}^{\beta^{\prime}}\left(u-u_{+}\right)(t, x)\right| \\
& \leq C e^{\mu\langle x\rangle / 4} \sum_{\left|\beta^{\prime}\right| \leq|\alpha|+\left|\alpha^{\prime}\right|}\left|\partial_{x}^{\beta^{\prime}}\left(u-u_{+}\right)(t, x)\right|
\end{aligned}
$$

with some positive constant $C=C\left(\alpha, \alpha^{\prime}, \mu\right)$. Similarly, if $j$ is odd, we get

$$
\left|\partial_{x}^{\alpha^{\prime}} Z^{\alpha}\left(u-u_{+}\right)(t, x)\right| \leq C e^{\mu\langle x\rangle / 4} \sum_{\left|\beta^{\prime}\right| \leq|\alpha|+\left|\alpha^{\prime}\right|-1}\left|\partial_{x}^{\beta^{\prime}} \partial_{t}\left(u-u_{+}\right)(t, x)\right| .
$$

Hence the left-hand side of (5.1) is bounded by

$$
C\left\|\exp (\mu\langle\cdot\rangle)\left(U(t) \vec{f}-\left.\left(U_{0}(t) \vec{f}_{+}\right)\right|_{\Omega}\right)\right\|_{\mathscr{H}^{[n / 2]+|\alpha|}(\Omega)},
$$

and (1.9) implies (5.1).

By (5.1) and the Sobolev imbedding theorem, we get

$$
\begin{aligned}
& \left|Z^{\alpha}\left(u-u_{+}\right)(t, x)\right| \\
& \leq C_{k} e^{-3 \mu\langle x\rangle / 4}\left\|e^{3 \mu\langle\cdot\rangle / 4} Z^{\alpha}\left(u-u_{+}\right)(t, \cdot)\right\|_{H^{[n / 2]+1}(\Omega)} \\
& \leq C_{k} e^{-3 \mu(t+\langle x\rangle) / 4}\|\vec{f}\|_{\mathscr{H}^{k+[n / 2]}(\Omega)} \\
& \leq C_{k}(1+t+|x|)^{-(n+1) / 2} e^{-\mu(t+|x|) / 2}\|\vec{f}\|_{\mathscr{H}^{k+[n / 2]}(\Omega)}
\end{aligned}
$$

for $|x| \geq t / 2 \geq 1$ and $|\alpha| \leq k$, where $C_{k}$ is a positive constant. Hence we find that our task is to show (1.12) and (1.13) with $u$ being replaced by $u_{+}$. Such estimates follow immediately from Proposition 4.1 with $v=0$ and $\vec{g}=\vec{f}_{+}$, since 
we have $\vec{f}_{+} \in\left(\mathscr{S}_{\chi}\left(\boldsymbol{R}^{n}\right)\right)^{2}$ with $\chi(s)=\exp (\mu s / 2)$ from (1.10). This completes the proof.

\title{
Acknowledgement
}

The authors would like to thank the referee for the useful comment. The research of the first author is partially supported by Grant-in-Aid for Scientific Research (C) (No. 20540211), JSPS. The research of the second author is partially supported by Grant-in-Aid for Science Research (No. 20224013), JSPS.

\section{References}

[ 1 ] Courant, R. and Hilbert, D., Methods of Mathematical Physics II, Interscience, New York, 1962.

[2] Friedlander, F. G., On the radiation field of pulse solutions of the wave equation, Proc. Roy. Soc. Ser. A, 269 (1962), 53-65.

[3] Friedlander, F. G., On the radiation field of pulse solutions of the wave equation. II, Proc. Roy. Soc. Ser. A, 279 (1964), 386-394.

[4] Friedlander, F. G., On the radiation field of pulse solutions of the wave equation. III, Proc. Roy. Soc. Ser. A, 299 (1967), 264-278.

[5] Friedlander, F. G., Radiation fields and hyperbolic scattering theory, Math. Proc. Cambridge Philos. Soc., 88 (1980), 483-515.

[6] Hörmander, L., Lectures on Nonlinear Hyperbolic Differential Equations, Springer-Verlag, Berlin, 1997.

[7] Ikawa, M., Hyperbolic Partial Differential Equations and Wave Phenomena, Translations of Mathematical Monographs 189, Iwanami Series in Modern Mathematics, AMS, Providence, RI, 2000.

[8] John, F., Existence for large times of strict solutions of nonlinear wave equations in three space dimensions for small initial data, Comm. Pure Appl. Math., 40 (1987), 79-109.

[9] Lax, P. D. and Phillips, R. S., Scattering Theory, Revised Edition, Academic Press, New York, 1989.

[10] Melrose, R. B., Singularities and energy decay in acoustical scattering, Duke Math. J., 46 (1979), 43-59.

[11] Shibata, Y. and Tsutsumi, Y., Global existence theorem for nonlinear wave equation in exterior domain, in "Recent topics in nonlinear PDE (Hiroshima, 1983)", 155-196, NorthHolland Math. Stud., 98, North-Holland, Amsterdam, 1984.

[12] Shibata, Y. and Tsutsumi, Y., On a global existence theorem of small amplitude solutions for nonlinear wave equations in an exterior domain, Math. Z., 191 (1986), 165-199.

[13] Katayama, S. and Kubo, H., Lower bound of the lifespan of solutions to semilinear wave equations in an exterior domain, preprint (arXiv:1009.1188[math.AP]).

\author{
nuna adreso: \\ Soichiro Katayama \\ Department of Mathematics \\ Wakayama University \\ 930 Sakaedani, Wakayama 640-8510 \\ Japan \\ E-mail: katayama@center.wakayama-u.ac.jp
}




\section{Hideo Kubo}

Division of Mathematics

Graduate School of Information Sciences

Tohoku University

Sendai 980-8579

Japan

E-mail: kubo@math.is.tohoku.ac.jp

(Ricevita la 16-an de majo, 2009)

(Reviziita la 9-an de aprilo, 2010) 